\title{
An Extremal Inequality Motivated by Multiterminal Information Theoretic Problems
}

\author{
Tie Liu and Pramod Viswanath *
}

November 30, 2018

\begin{abstract}
We prove a new extremal inequality, motivated by the vector Gaussian broadcast channel and the distributed source coding with a single quadratic distortion constraint problem. As a corollary, this inequality yields a generalization of the classical vector entropy-power inequality (EPI). As another corollary, this inequality sheds insight into maximizing the differential entropy of the sum of two jointly distributed random variables.
\end{abstract}

Keywords: Differential entropy, distributed source coding, entropy-power inequality (EPI), Fisher information inequality (FII), vector Gaussian broadcast channel

\section{Introduction}

Like many other important results in information theory, the classical entropy-power inequality (EPI) was discovered by Shannon [1] (even though the first rigorous proof was established by Stam [2] and was later simplified by Blachman [3]). In [1, p. 641], Shannon used the classical EPI to prove a lower bound on the capacity of arbitrary additive noise channels. While the first application was on a point-to-point scenario, the real utility of EPI showed up much later in the multiterminal source/channel coding problems where the tension among users

${ }^{*}$ The authors are with the Department of Electrical and Computer Engineering at the University of Illinois at Urbana-Champaign, Urbana, IL 61801, USA (e-mail: tieliu@ifp.uiuc.edu; pramodv@uiuc.edu). 
of different interests cannot be resolved by Fano's inequality alone. The most celebrated examples include Bergman's solution to the scalar Gaussian broadcast channel problem [4, Oohama's solution to the quadratic scalar Gaussian CEO problem [5], and Ozarow's solution to the scalar Gaussian two-description problem [6].

Denote the set of real numbers by $\mathcal{R}$. Let $\mathbf{X}$ and $\mathbf{Z}$ be two independent random vectors with densities in $\mathcal{R}^{n}$. The classical EPI can be stated as:

$$
e^{2 h(\mathbf{X}+\mathbf{Z}) / n} \geq e^{2 h(\mathbf{X}) / n}+e^{2 h(\mathbf{Z}) / n}
$$

where $h(\cdot)$ denotes the differential entropy of the corresponding random vector, and the equality holds if and only if $\mathbf{X}$ and $\mathbf{Z}$ are Gaussian with proportional covariance matrices.

Fix $\mathbf{Z}$ to be a Gaussian vector with a strictly positive definite covariance matrix $\mathbf{K}_{Z}$. Consider the optimization problem:

$$
\max _{\mathbf{X}} h(\mathbf{X})-\mu h(\mathbf{X}+\mathbf{Z})
$$

where $\mu$ is a real number greater than one, and the maximization is over all random vectors independent of $\mathbf{Z}$. The classical vector EPI can be used to show that an optimal solution to the optimization problem (2) is a vector Gaussian $\mathbf{X}_{G}^{*}$ with a covariance matrix proportional to $\mathbf{K}_{Z}$. This can be done as follows:

$$
\begin{aligned}
h(\mathbf{X})-\mu h(\mathbf{X}+\mathbf{Z}) & \leq h(\mathbf{X})-\frac{\mu n}{2} \log _{e}\left(e^{2 h(\mathbf{X}) / n}+e^{2 h(\mathbf{Z}) / n}\right) \\
& \leq h\left(\mathbf{X}_{G}^{*}\right)-\frac{\mu n}{2} \log _{e}\left(e^{2 h\left(\mathbf{X}_{G}^{*}\right) / n}+e^{2 h(\mathbf{Z}) / n}\right)
\end{aligned}
$$

where $h\left(\mathbf{X}_{G}^{*}\right)=h(\mathbf{Z})-(n / 2) \log _{e}(\mu-1)$, (3) follows from the classical vector EPI, and (4) follows from the fact that for $\mu>1$

$$
f(t)=t-\frac{\mu n}{2} \log _{e}\left(e^{2 t / n}+e^{2 a / n}\right)
$$

is concave in $\mathcal{R}$ with a global maxima at

$$
t=a-(n / 2) \log _{e}(\mu-1) .
$$

Checking the equality conditions of (3) and (4), we conclude that the optimization problem (2) has a Gaussian optimal solution $\mathbf{X}_{G}^{*}$ with a covariance matrix proportional to $\mathbf{K}_{Z}$.

Note that in solving the optimization problem (2), the use of the classical vector EPI not only forces the optimal solution to be Gaussian but also imposes a certain covariance structure on the Gaussian optimal solution. A natural question to ask is what happens if there exists an extra covariance constraint on $\mathbf{X}$ such that the original optimal solution is no longer admissible. In that case, the classical vector EPI may still be used as in (3); however, 
the equality may no longer be achieved because the new optimal solution may no longer have the required proportionality. Particularly, one would be interested in finding out whether, under the additional covariance constraint, the optimization problem (2) still has a Gaussian optimal solution.

One particular type of covariance constraint is the following power-covariance type constraint:

$$
\operatorname{Cov}(\mathbf{X}) \preceq \mathbf{S}
$$

where $\operatorname{Cov}(\cdot)$ denotes the covariance matrix of the corresponding random vector, “ $\preceq$ " represents "less or equal than" in the positive semidefinite partial ordering of real symmetric matrices, and $\mathbf{S}$ is an arbitrary positive semidefinite matrix. The reason for considering such power-covariance type of constraint is largely due to its generality: it subsumes many other covariance constraints including the trace constraint.

The focus of the paper is the following slightly more general optimization problem:

$$
\begin{array}{ll}
\max _{\mathbf{X}} & h\left(\mathbf{X}+\mathbf{Z}_{1}\right)-\mu h\left(\mathbf{X}+\mathbf{Z}_{2}\right) \\
\text { subject to } & \operatorname{Cov}(\mathbf{X}) \preceq \mathbf{S}
\end{array}
$$

where $\mathbf{Z}_{1}$ and $\mathbf{Z}_{2}$ are Gaussian vectors with strictly positive definite covariance matrices $\mathbf{K}_{Z_{1}}$ and $\mathbf{K}_{Z_{2}}$, respectively, $\mu$ is a real number, $\mathbf{S}$ is an arbitrary positive semidefinite matrix, and the maximization is over all random vectors $\mathbf{X}$ independent of $\mathbf{Z}_{1}$ and $\mathbf{Z}_{2}$. As we shall see, such an optimization problem appears naturally when one is to seek the tightest outer bound of a certain genie-aided type (cf. Theorem 5 of [7]) for the capacity/rate region of the vector Gaussian broadcast channel and the distributed source coding with a single quadratic distortion constraint problem. The main result of this paper is summarized in the following theorem.

Theorem 1 The optimization problem (8) has a Gaussian optimal solution for all $\mu \geq 1$.

The rest of the paper is organized as follows. In Section 2, we establish the connections between the optimization problem (8) and the vector Gaussian broadcast channel and the distributed source coding with a single quadratic distortion constraint problem; these connections are the main motivations of this paper. The proof of the main result is in Section 3 . Two proofs will be given: a direct proof using the classical vector EPI, and a strengthened proof using the more traditional perturbation approach. The implications of the main result are discussed in Section 4. Finally in Section 5, we conclude by summarizing our contribution in the context of the applications of information theoretic inequalities in resolving multiterminal transmission/compression problems. 


\section{Motivations from Multiterminal Information Theory}

\subsection{The Vector Gaussian Broadcast Channel}

Consider the following two-user discrete memoryless vector Gaussian broadcast channel:

$$
\mathbf{Y}_{i}[k]=\mathbf{X}[k]+\mathbf{Z}_{i}[k], \quad i=1,2 ; \quad k=1, \cdots, n
$$

where $\{\mathbf{X}[k]\}_{k=1}^{n}$ is the channel input subject to the constraint:

$$
\frac{1}{n} \sum_{k=1}^{n} \mathbf{X}[k] \mathbf{X}^{t}[k] \preceq \mathbf{S}
$$

$\left\{\mathbf{Z}_{i}[k]\right\}_{k=1}^{n}$ is an independent Gaussian noise process with strictly positive definite covariance matrix $\mathbf{K}_{Z_{i}}$ for each component, and $\{\mathbf{X}[k]\}_{k=1}^{n}$ and $\left\{\mathbf{Z}_{i}[k]\right\}_{k=1}^{n}$ are statistically independent. The covariance structure of individual vector symbols models a scalar Gaussian broadcast channel with arbitrary stationary Gaussian noise processes. Alternatively, it can be used to model the the downlink of a cellular system with multiple antennas; this was the motivation of the authors in 9], where the covariance matrices $\mathbf{K}_{Z_{i}}, i=1,2$, are used to model the effects of multiplexing of different transmission paths from multiple transmit antennas to multiple receive antennas.

The capacity region of the vector Gaussian broadcast channel had been a long-standing open problem in multiterminal information theory (particularly when viewed in the context of a scalar Gaussian broadcast channel with arbitrary stationary Gaussian noise processes). Before [9], only certain bounds are known. The best known outer bound, derived by Marton and Körner [7, Theorem 5], can be written as:

$$
\begin{aligned}
& R_{1} \leq I\left(\mathbf{X} ; \mathbf{Y}_{1} \mid U\right)=h\left(\mathbf{X}+\mathbf{Z}_{1} \mid U\right)-h\left(\mathbf{Z}_{1}\right) \\
& R_{2} \leq I\left(U ; \mathbf{Y}_{2}\right)=h\left(\mathbf{Y}_{2}\right)-h\left(\mathbf{X}+\mathbf{Z}_{2} \mid U\right)
\end{aligned}
$$

for any $(U, \mathbf{X})$ satisfying:

1. $U \leftrightarrow \mathbf{X} \leftrightarrow\left(\mathbf{Y}_{1}, \mathbf{Y}_{2}\right)$ forms a Markov chain;

2. $\mathbb{E}\left[\mathbf{X X}^{t}\right] \preceq \mathbf{S}$.

It is also known that if $(\mathbf{X}, U)$ are jointly Gaussian, the outer region (111) coincides with an achievable region called the Costa region. So if we can show that the largest outer region (as that of Marton and Körner) is given by a jointly Gaussian $(\mathbf{X}, U)$, we will have an exact characterization for the capacity region of the vector Gaussian broadcast channel (9). 
To that end let us consider maximizing the weighted sum rate

$$
R_{1}+\mu R_{2} \leq \mu h\left(\mathbf{Y}_{2}\right)-h\left(\mathbf{Z}_{1}\right)+h\left(\mathbf{X}+\mathbf{Z}_{1} \mid U\right)-\mu h\left(\mathbf{X}+\mathbf{Z}_{2} \mid U\right)
$$

Here we focus on $\mu \geq 1$, because the other outer region of Marton and Körner ${ }^{1}$ is strictly smaller for $0 \leq \mu<1$. Under the constraint $\mathbb{E}\left[\mathbf{X} \mathbf{X}^{t}\right] \preceq \mathbf{S}, h\left(\mathbf{Y}_{2}\right)$ is maximized when $\mathbf{X}$ is Gaussian with zero mean and covariance matrix $\mathbf{S}$. Therefore, we may consider separately maximizing $h\left(\mathbf{Y}_{2}\right)$ and

$$
h\left(\mathbf{X}+\mathbf{Z}_{1} \mid U\right)-\mu h\left(\mathbf{X}+\mathbf{Z}_{2} \mid U\right)=\int\left(h\left(\mathbf{X}+\mathbf{Z}_{1} \mid U=u\right)-\mu h\left(\mathbf{X}+\mathbf{Z}_{2} \mid U=u\right)\right) d P_{U}(u) .
$$

Since $\mathbf{Z}_{1}$ and $\mathbf{Z}_{2}$ are independent of $U$ and the constraint $\mathbb{E}\left[\mathbf{X} \mathbf{X}^{t}\right] \preceq \mathbf{S}$ can be rewritten as

$$
\int \mathbb{E}\left[\mathbf{X X}^{t} \mid U=u\right] d P_{U}(u) \preceq \mathbf{S}
$$

the second optimization problem is nothing but an average version of (8) where the averaging is over $U$. Because of our separate-maximization strategy, Theorem 1 is potentially stronger than the broadcast channel result.

On the other hand, we note that Theorem 1 alone is not enough to prove the desired broadcast channel result. To complete the argument, we need to show that the value of the optimization problem (8) is a concave function of the parameter $\mathbf{S}$ in the constraint. Unfortunately, so far we have neither been able to prove nor disapprove this conjecture.

\subsection{Distributed Source Coding with a Single Quadratic Distortion Constraint}

Consider the following distributed source coding problem with a single quadratic distortion constraint: $\left\{\mathbf{Y}_{1}[k]\right\}_{k=1}^{n}$ and $\left\{\mathbf{Y}_{2}[k]\right\}_{k=1}^{n}$ are two discrete memoryless vector sources. For each $k, \mathbf{Y}_{1}[k]$ and $\mathbf{Y}_{2}[k]$ are jointly Gaussian vectors with strictly positive definite covariance matrices $\mathbf{K}_{Y_{1}}$ and $\mathbf{K}_{Y_{2}}$, respectively. (The covariance matrices are used to model the inherent memory in the sources.) The encoder is only allowed to perform separate encoding on $\left\{\mathbf{Y}_{1}[k]\right\}_{k=1}^{n}$ and $\left\{\mathbf{Y}_{2}[k]\right\}_{k=1}^{n}$. On the other hand, the purpose of the decoder is to reconstruct $\left\{\mathbf{Y}_{1}[k]\right\}_{k=1}^{n}$ based on both encoded messages. The question is what is the achievable rate region for the encoders such that the mean-squared error distortion for reconstructing $\left\{\mathbf{Y}_{1}[k]\right\}_{k=1}^{n}$ at the decoder is no greater than a specified number $D$.

\footnotetext{
${ }^{1}$ For each given $(\mathbf{X}, U)$, there are two regions of the Marton-Körner-type associated with a two-user discrete memoryless broadcast channel. The one described by (11) is obtained by giving the message intended for User 2 to User 1 while decoding his own message.
} 
Even though the capacity region for this problem remains unknown, there exists a natural separation scheme which can be described as follows. First, quantize $\left\{\mathbf{Y}_{1}[k]\right\}_{k=1}^{n}$ and $\left\{\mathbf{Y}_{2}[k]\right\}_{k=1}^{n}$ separately using Gaussian codebooks. Then, perform Slepian-Wolf [8] coding on the quantized version of $\left\{\mathbf{Y}_{1}[k]\right\}_{k=1}^{n}$ by treating the quantized version of $\left\{\mathbf{Y}_{2}[k]\right\}_{k=1}^{n}$ as side information available to the decoder only. The rate region achieved by such a Gaussian separation scheme is given by:

$$
\begin{aligned}
& R_{1} \leq I\left(\mathbf{Y}_{1} ; \widehat{\mathbf{Y}}_{1} \mid U\right)=h\left(\mathbf{Y}_{1} \mid U\right)-h\left(\mathbf{Y}_{1} \mid \widehat{\mathbf{Y}}_{1}, U\right) \\
& R_{2} \leq I\left(U ; \mathbf{Y}_{2}\right)=h\left(\mathbf{Y}_{2}\right)-h\left(\mathbf{Y}_{2} \mid U\right)
\end{aligned}
$$

where $\left(U, \widehat{\mathbf{Y}}_{1}\right)$ is jointly Gaussian with $\left(\mathbf{Y}_{1}, \mathbf{Y}_{2}\right)$ and satisfies:

1. $\widehat{\mathbf{Y}}_{1} \leftrightarrow U \leftrightarrow \mathbf{Y}_{2}$ forms a Markov chain;

2. $\mathbb{E}\left[\left(\mathbf{Y}_{1}-\widehat{\mathbf{Y}}_{1}\right)^{t}\left(\mathbf{Y}_{1}-\widehat{\mathbf{Y}}_{1}\right)\right] \leq D$.

Naturally, one may wonder whether the rate region (15) is the true rate region for this problem.

From the converse viewpoint, we can show that, without assuming that $\left(U, \widehat{\mathbf{Y}}_{1}\right)$ is jointly Gaussian with $\left(\mathbf{Y}_{1}, \mathbf{Y}_{2}\right)$, the right-hand sides of (15) are in fact an outer bound for the rate region of the distributed source coding with a single quadratic distortion constraint problem. (The details of the proof are in Appendix [A.) This is analogous to Marton and Körner's outer bound for the capacity region of the discrete memoryless broadcast channel. Thus, to show the tightness of the rate region described by (15), it is sufficient to show that the right-hand sides of (15) are minimized by a $\left(U, \widehat{\mathbf{Y}}_{1}\right)$ that is jointly Gaussian with $\left(\mathbf{Y}_{1}, \mathbf{Y}_{2}\right)$.

Similarly to the broadcast channel problem, we consider minimizing the weighted sum rate

$$
\mu R_{1}+R_{2} \geq \mu h\left(\mathbf{Y}_{1} \mid U\right)-\mu h\left(\mathbf{Y}_{1} \mid \tilde{\mathbf{Y}}_{1}, U\right)+h\left(\mathbf{Y}_{2}\right)-h\left(\mathbf{Y}_{2} \mid U\right)
$$

for $\mu \geq 1$. $h\left(\mathbf{Y}_{2}\right)$ is a constant, and $h\left(\mathbf{Y}_{1} \mid \widehat{\mathbf{Y}}_{1}, U\right)$ can be bounded from above as:

$$
h\left(\mathbf{Y}_{1} \mid \widehat{\mathbf{Y}}_{1}, U\right)=h\left(\mathbf{Y}_{1}-\widehat{\mathbf{Y}}_{1} \mid \widehat{\mathbf{Y}}_{1}, U\right) \leq h\left(\mathbf{Y}_{1}-\widehat{\mathbf{Y}}_{1}\right)
$$

Under the quadratic distortion constraint $\mathbb{E}\left[\left(\mathbf{Y}_{1}-\widehat{\mathbf{Y}}_{1}\right)^{t}\left(\mathbf{Y}_{1}-\widehat{\mathbf{Y}}_{1}\right)\right] \leq D$, the right-hand side of (17) is maximized when $\mathbf{Y}_{1}-\widehat{\mathbf{Y}}_{1}$ is vector Gaussian with identical variance for each component. So we only need to consider maximizing

$$
h\left(\mathbf{Y}_{2} \mid U\right)-\mu h\left(\mathbf{Y}_{1} \mid U\right)=\int\left(h\left(\mathbf{Y}_{2} \mid U=u\right)-\mu h\left(\mathbf{Y}_{1} \mid U=u\right)\right) d P_{U}(u)
$$

subject to the constraints

$$
\operatorname{Cov}\left(\mathbf{Y}_{2} \mid U\right) \preceq \operatorname{Cov}\left(\mathbf{Y}_{2}\right) \text { and } \operatorname{Tr}\left(\operatorname{Cov}\left(\mathbf{Y}_{1} \mid U\right)\right) \geq D .
$$


Now rewriting the first constraint as

$$
\int \operatorname{Cov}\left(\mathbf{Y}_{2} \mid U=u\right) d P_{U}(u) \preceq \operatorname{Cov}\left(\mathbf{Y}_{2}\right)
$$

and ignoring the second constraint ${ }^{2}$, the optimization problem (18) is once again an average version of (8).

\section{Proofs of the Main Result}

\subsection{A Direct Proof}

We first show that the classical vector EPI can be appropriately used to give a direct proof to Theorem 1, which is the main result of the paper. That the classical vector EPI is involved is barely surprising, considering that the objective function of the optimization problem (8) involves the entropy of the sum of two independent random vectors. Nevertheless, based on the discussion in Section 11, a direct use of the classical vector EPI might be loose because the covariance matrix of the optimal solution may not have the required proportionality.

The way we resolve this issue is inspired by the mathematical import of an interesting technique called enhancing from Weingarten, Steinberg and Shamai [9], where it was key to their proof of the converse coding theorem for the vector Gaussian broadcast channel problem. Our proof combines the idea of enhancing with the worst additive noise lemma, which was independently proved by Ihara [12 and by Diggavi and Cover [13, Lemma II.2]. The details of the proof are deferred to Appendix B.

\subsection{An Isoperimetric View}

From an optimization point of view, the power of the classical EPI roots in its ability to find global optima in nonconvex programs such as (2). One can thus imagine that the proof of the classical EPI cannot be accomplished by any local optimization technique. In their proof Stam [2] and Blachman [3] used a perturbation technique, which amounts to finding a monotone path from any distributions of the participating random vectors to the optimal distributions for which the classical EPI holds with equality. The monotonicity guarantees that any distributions along the path satisfy the desired inequality, and hence so do the ones

\footnotetext{
${ }^{2}$ Unfortunately, there are some instances where it cannot be ignored; in such cases, the outer bound studied here will be strictly inside the inner bound achieved by the natural Gaussian-separation scheme.
} 
to begin with. A different perturbation was later given by Dembo, Cover and Thomas [10]. The main idea, however, is the same as that of Stam and Blachman's.

Proving monotonicity needs isoperimetric inequalities and, in case of the classical EPI, the classical Fisher information inequality (FII) [10, Theorem 13]. Fisher information is an important quantity in estimation theory. An interesting estimation theoretic proof of the classical FII was given by Zamir [14]. ${ }^{3}$ This connection between the classical EPI and FII is usually thought of as the estimation view of the classical EPI.

We may use the perturbation idea to give a stronger proof to Theorem 10 In particular, we may construct a monotone path using the "covariance-preserving" transformation, which was previously used by Dembo, Cover and Thomas [10, Section III-C] in their perturbation proof of the classical EPI. (The details of the proof are in Appendix C) To demonstrate the monotonicity, we prove the following novel monotonicity result of Fisher information as an addition to the classical FII.

Lemma $\mathbf{2}$ Let $\mathbf{Z}$ and $\widetilde{\mathbf{Z}}$ be two Gaussian vectors with positive definite covariance matrix $\mathbf{K}_{Z}$ and $\widetilde{\mathbf{K}}_{Z}$, respectively and assume that $\mathbf{K}_{Z} \succeq \widetilde{\mathbf{K}}_{Z}$. Denote by $\mathbf{J}(\cdot)$ the Fisher information matrix of the corresponding random vector. We have

$$
\operatorname{Tr}(\mathbf{A} \mathbf{J}(\mathbf{X}+\mathbf{Z})) \leq \operatorname{Tr}(\widetilde{\mathbf{A}} \mathbf{J}(\mathbf{X}+\widetilde{\mathbf{Z}}))
$$

for any positive definite matrices $\mathbf{A}$ and $\widetilde{\mathbf{A}}$ satisfying:

1. $\mathbf{A} \succeq \widetilde{\mathbf{A}}$

2. $\mathbf{A}^{-1}\left(\mathbf{K}_{X}+\mathbf{K}_{Z}\right)=\widetilde{\mathbf{A}}^{-1}\left(\mathbf{K}_{X}+\mathbf{K}_{\widetilde{Z}}\right)$

and any random vector $\mathbf{X}$ with covariance matrix $\mathbf{K}_{X}$ and independent of $\mathbf{Z}$ and $\widetilde{\mathbf{Z}}$. In particular, we have

$$
\operatorname{Tr}\left(\left(\mathbf{K}_{X}+\mathbf{K}_{Z}\right) \mathbf{J}(\mathbf{X}+\mathbf{Z})\right) \leq \operatorname{Tr}\left(\left(\mathbf{K}_{X}+\mathbf{K}_{\widetilde{Z}}\right) \mathbf{J}(\mathbf{X}+\widetilde{\mathbf{Z}})\right)
$$

This monotonicity result of Fisher information can be understood via the following consequence of the Cramér-Rao inequality [10, Theorem 20]:

$$
\operatorname{Tr}\left(\mathbf{K}_{X} \mathbf{J}(\mathbf{X})\right) \geq n
$$

\footnotetext{
${ }^{3}$ The classical FII can also be proved by using the standard data processing inequality for mutual information, invoking a connection between Fisher information and the derivative of mutual information explicitly established by Guo, Shamai and Verdú [15, Corollary 2].
} 
where $n$ is the dimension of $\mathbf{X}$, and the equality holds if and only if $\mathbf{X}$ is Gaussian. This inequality suggests that we may use $\operatorname{Tr}\left(\mathbf{K}_{X} \mathbf{J}(\mathbf{X})\right)$ as a measure of the Gaussianity of $\mathbf{X}$ : the smaller $\operatorname{Tr}\left(\mathbf{K}_{X} \mathbf{J}(\mathbf{X})\right)$ is, the more Gaussian $\mathbf{X}$ is. Lemma 2 provides a proper justification for such intuition by proving the monotonicity of $\operatorname{Tr}\left(\left(\mathbf{K}_{X}+\mathbf{K}_{Z}\right) \mathbf{J}(\mathbf{X}+\mathbf{Z})\right)$ with respect to the proportion of the Gaussian component $\mathbf{Z}$. For the scalar case, Lemma 2 can be proved by combining the classical FII and the Cramér-Rao inequality. For the general vector case, however, we cannot quite do that. Instead, we resort to a method that relies on several important properties of score function. Some preliminaries on the Fisher information and the score function as well as the proof of Lemma 2 are presented in Appendix D.

\section{Implications of the Main Result}

Two special cases of the general optimization problem (8) demonstrate the breadth of our main result; we term these two scenarios as the degraded case and the extremely-skewed case. By considering the degraded case, we prove a generalization of the classical vector EPI; by considering the extremely-skewed case, we establish a connection between our main result and the classical result of Cover and Zhang [11] on the maximum differential entropy of the sum of two jointly distributed random variables.

\subsection{A Generalization of the Classical Vector EPI}

In the degraded case, we have either $\mathbf{K}_{Z_{1}} \preceq \mathbf{K}_{Z_{2}}$ or $\mathbf{K}_{Z_{1}} \succeq \mathbf{K}_{Z_{2}}$. Let us first consider the case where $\mathbf{K}_{Z_{1}} \preceq \mathbf{K}_{Z_{2}}$. We may write $\mathbf{Z}_{2}=\mathbf{Z}_{1}+\mathbf{Z}$ where $\mathbf{Z}$ is a Gaussian vector independent of $\mathbf{Z}_{1}$. We have the following corollaries.

Corollary 3 Let $\mathbf{Z}_{1}$ and $\mathbf{Z}$ be two independent Gaussian vectors with covariance matrices $\mathbf{K}_{Z_{1}}$ and $\mathbf{K}_{Z}$, respectively. Assume that $\mathbf{K}_{Z_{1}}$ is strictly positive definite. The optimization problem

$$
\begin{array}{ll}
\max _{\mathbf{X}} & h\left(\mathbf{X}+\mathbf{Z}_{1}\right)-\mu h\left(\mathbf{X}+\mathbf{Z}_{1}+\mathbf{Z}\right) \\
\text { subject to } & \operatorname{Cov}(\mathbf{X}) \preceq \mathbf{S}
\end{array}
$$

where $\mathbf{S}$ is an arbitrary semidefinite matrix, and the maximization is over all random vectors independent of $\mathbf{Z}_{1}$ and $\mathbf{Z}$ has a Gaussian optimal solution for all $\mu \in \mathcal{R}$.

Proof: For $\mu \geq 1$, the corollary follows directly from Theorem 1 by letting $\mathbf{Z}_{2}=\mathbf{Z}_{1}+\mathbf{Z}$. The corollary is also true for $\mu \leq 0$, because $h\left(\mathbf{X}+\mathbf{Z}_{1}\right)$ and $h\left(\mathbf{X}+\mathbf{Z}_{1}+\mathbf{Z}\right)$ are simultaneously 
maximized when $\mathbf{X}$ is Gaussian with covariance matrix $\mathbf{S}$. This leaves us the only case where $0<\mu<1$.

Note that the objective function of the optimization problem (24) can be written as:

$$
(1-\mu) h\left(\mathbf{X}+\mathbf{Z}_{1}\right)+\mu\left(h\left(\mathbf{X}+\mathbf{Z}_{1}\right)-h\left(\mathbf{X}+\mathbf{Z}_{1}+\mathbf{Z}\right)\right) .
$$

It is known that $h\left(\mathbf{X}+\mathbf{Z}_{1}\right)$ is maximized when $\mathbf{X}$ is Gaussian with full covariance matrix $\mathbf{S}$. By the worst additive noise result of Lemma $\mathbf{8}, h\left(\mathbf{X}+\mathbf{Z}_{1}\right)-h\left(\mathbf{X}+\mathbf{Z}_{1}+\mathbf{Z}\right)$ is also maximized when $\mathbf{X}$ is Gaussian. Within the Gaussian class, it is clear that a vector Gaussian $\mathbf{X}$ with full covariance matrix $\mathbf{S}$ minimizes $I\left(\mathbf{Z} ; \mathbf{Z}+\mathbf{X}+\mathbf{Z}_{1}\right)$ and hence maximizes $h\left(\mathbf{X}+\mathbf{Z}_{1}\right)-$ $h\left(\mathbf{X}+\mathbf{Z}_{1}+\mathbf{Z}\right)$. For $\mu \in(0,1)$, both $\mu$ and $1-\mu$ are positive. We may conclude that (25) is maximized when $\mathbf{X}$ is Gaussian with covariance matrix $\mathbf{S}$. This completes the proof of Corollary 3 .

Corollary 4 Let $\mathbf{Z}$ be a Gaussian vector with covariance matrix $\mathbf{K}_{Z}$ and assume that $\mathbf{K}_{Z}$ is strictly positive definite. The optimization problem

$$
\begin{array}{ll}
\max _{\mathbf{X}} & h(\mathbf{X})-\mu h(\mathbf{X}+\mathbf{Z}) \\
\text { subject to } & \operatorname{Cov}(\mathbf{X}) \preceq \mathbf{S}
\end{array}
$$

where $\mathbf{S}$ is an arbitrary semidefinite matrix, and the maximization is over all random vectors independent of $\mathbf{Z}$ has a Gaussian optimal solution for all $\mu \in \mathcal{R}$.

Note that the optimization problem (26) is a constrained version of the optimization problem (2). Recall from Section 1 that the optimization problem (2) can be solved by the classical EPI. Conversely, we may also deduce a special case of the classical EPI (where one of the participating random vector is fixed to be Gaussian) from the fact that the optimization problem (2) has a Gaussian optimal solution. This can be done as follows: for any random vector $\mathbf{X}$ independent of $\mathbf{Z}$, we may choose $\mu=1+\exp (2(h(\mathbf{Z})-h(\mathbf{X})) / n)$. With this choice of $\mu$, we have $h\left(\mathbf{X}_{G}^{*}\right)=h(\mathbf{X})$ where $\mathbf{X}_{G}^{*}$ is the optimal Gaussian solution of the optimization problem (2). By the fact that $\mathbf{X}_{G}^{*}$ is also the optimal solution of the optimization problem (2), we have $h(\mathbf{X})-\mu h(\mathbf{X}+\mathbf{Z}) \leq h\left(\mathbf{X}_{G}^{*}\right)-\mu h\left(\mathbf{X}_{G}^{*}+\mathbf{Z}\right)$ for any random vector $\mathbf{X}$ independent of $\mathbf{Z}$. Combined with $h\left(\mathbf{X}_{G}^{*}\right)=h(\mathbf{X})$, we obtain that $h(\mathbf{X}+\mathbf{Z}) \geq h\left(\mathbf{X}_{G}^{*}+\mathbf{Z}\right)$ for any random vector $\mathbf{X}$ independent of $\mathbf{Z}$ and satisfying $h(\mathbf{X})=h\left(\mathbf{X}_{G}^{*}\right)$, which is precisely the Costa-Cover form of the classical EPI [10, Theorem 6]. In this sense, Corollary 4 can be thought of as a generalization of (a special case of) the classical vector EPI.

For technical reasons, Corollary 4 cannot be proved by letting the covariance matrix of $\mathbf{Z}_{1}$ in Corollary 3 vanish. Instead, it has to be proved in a manner similar to that for Theorem 1. The details of the proofs (the direct one and the perturbation one) are omitted from the paper due to their similarities to those of Theorem 1] 
We now turn to the other degraded case where $\mathbf{K}_{Z_{1}} \succeq \mathbf{K}_{Z_{2}}$. We may write $\mathbf{Z}_{1}=\mathbf{Z}_{2}+\mathbf{Z}$ where $\mathbf{Z}$ is a Gaussian vector with covariance matrix $\mathbf{K}_{Z}$ and independent of $\mathbf{Z}_{2}$. Consider the optimization problem:

$$
\begin{array}{ll}
\max _{\mathbf{X}} & h\left(\mathbf{X}+\mathbf{Z}_{2}+\mathbf{Z}\right)-\mu h\left(\mathbf{X}+\mathbf{Z}_{2}\right) \\
\text { subject to } & \operatorname{Cov}(\mathbf{X}) \preceq \mathbf{S}
\end{array}
$$

where $\mathbf{S}$ is an arbitrary positive semidefinite matrix, and the maximization is over all random vectors independent of $\mathbf{Z}_{2}$ and $\mathbf{Z}$. Theorem $\mathbf{1}$ asserts that the optimization problem (27) has a Gaussian optimal solution for all $\mu \geq 1$. Clearly, this is also true for $\mu \leq 0$. However, as we shall see next, this is generally not true for $0<\mu<1$.

Let $\mathbf{K}_{X}^{*}:=\frac{\mu}{1-\mu} \mathbf{K}_{Z}-\mathbf{K}_{Z_{2}}$ and assume that $\mathbf{K}_{X}^{*}$ is strictly positive definite and strictly less than $\mathbf{S}$. Denote by $\mathbf{X}_{G}^{*}$ the optimal Gaussian solution to the optimization problem (27). Under the assumption, we can verify that the covariance matrix of $\mathbf{X}_{G}^{*}$ must be equal to $\mathbf{K}_{X}^{*}$. Let $\mathbf{X}$ be a non-Gaussian random vector satisfying $h\left(\mathbf{X}+\mathbf{Z}_{2}\right)=h\left(\mathbf{X}_{G}^{*}+\mathbf{Z}_{2}\right)$ and $\operatorname{Cov}(\mathbf{X}) \preceq \mathbf{S}$. Such $\mathbf{X}$ exists because by the assumption, the covariance matrix of $\mathbf{X}_{G}^{*}$ is strictly between $\mathbf{0}$ and $\mathbf{S}$. Since $\mathbf{X}$ is non-Gaussian, by the classical EPI, we have $h\left(\mathbf{X}+\mathbf{Z}_{2}+\mathbf{Z}\right)>h\left(\mathbf{X}_{G}^{*}+\mathbf{Z}_{2}+\mathbf{Z}\right)$ with the inequality being strict. We thus conclude that at least for this case, the optimal Gaussian solution $\mathbf{X}_{G}^{*}$ cannot be an optimal solution of the optimization problem (27).

\subsection{The Maximum Differential Entropy of the Sum of Two Jointly Distributed Random Variables}

Assume that $\mathbf{Z}_{1}$ and $\mathbf{Z}_{2}$ are in $\mathcal{R}^{2}$. The eigenvalue decompositions of $\mathbf{K}_{Z_{1}}$ and $\mathbf{K}_{Z_{2}}$ can be written as:

$$
\mathbf{K}_{Z_{1}}=\mathbf{V}_{1} \Sigma_{1} \mathbf{V}_{1}^{t}, \quad \mathbf{K}_{Z_{2}}=\mathbf{V}_{2} \Sigma_{2} \mathbf{V}_{2}^{t}
$$

where $\mathbf{V}_{1}, \mathbf{V}_{2}$ are $2 \times 2$ orthonormal matrices and $\boldsymbol{\Sigma}_{1}=\operatorname{Diag}\left(\lambda_{11}, \lambda_{12}\right), \boldsymbol{\Sigma}_{2}=\operatorname{Diag}\left(\lambda_{21}, \lambda_{22}\right)$ are $2 \times 2$ diagonal matrices. Consider the limiting situation where $\lambda_{12}, \lambda_{21} \rightarrow \infty$ while $\lambda_{11}$ and $\lambda_{22}$ are fixed. Compared with the degraded case where $\mathbf{K}_{Z_{1}}$ dominates $\mathbf{K}_{Z_{2}}$ in every direction (or vice versa), this situation between $\mathbf{K}_{Z_{1}}$ and $\mathbf{K}_{Z_{2}}$ is extremely skewed. We have the following corollary.

Corollary $\mathbf{5}$ Let $Z$ be a Gaussian variable with strictly positive covariance, and let $\mathbf{v}_{1}, \mathbf{v}_{2}$ be two deterministic vectors in $\mathcal{R}^{2}$. The optimization problem

$$
\begin{array}{ll}
\max _{\mathbf{X}} & h\left(\mathbf{v}_{1}^{t} \mathbf{X}+Z\right)-\mu h\left(\mathbf{v}_{2}^{t} \mathbf{X}+Z\right) \\
\text { subject to } & \operatorname{Cov}(\mathbf{X}) \preceq \mathbf{S}
\end{array}
$$

where $\mathbf{S}$ is an arbitrary $2 \times 2$ semidefinite matrix, and the maximization is over all random vectors in $\mathcal{R}^{2}$ independent of $Z$ has a Gaussian optimal solution for all $\mu \geq 1$. 
The proof is deferred to Appendix E Next, we use Corollary 5 to solve an optimization problem that involves maximizing the differential entropy of the sum of two jointly distributed variables. To put in perspective, let us first consider the following optimization problem:

$$
\begin{array}{ll}
\max _{X_{1}, X_{2}} & h\left(X_{1}+X_{2}\right) \\
\text { subject to } & \operatorname{Var}\left(X_{1}\right) \leq a_{1}, \quad \operatorname{Var}\left(X_{2}\right) \leq a_{2}
\end{array}
$$

where $a_{1}, a_{2}$ are two arbitrary nonnegative real numbers, $\operatorname{Var}(\cdot)$ denotes the variance of the corresponding random variable, and the maximization is over all jointly distributed random variables. Clearly, given the problem setup, $h\left(X_{1}+X_{2}\right)$ is maximized when $X_{1}$ and $X_{2}$ are jointly Gaussian with variance $a_{1}$ and $a_{2}$, respectively and are aligned, i.e., $X_{1}=\sqrt{a_{1} / a_{2}} X_{2}$ almost surely.

Substituting the variance constraints in the optimization problem (30) by entropy constraints, we have the following new optimization problem:

$$
\begin{array}{ll}
\max _{X_{1}, X_{2}} & h\left(X_{1}+X_{2}\right) \\
\text { subject to } & h\left(X_{1}\right) \leq a_{1}, \quad h\left(X_{2}\right) \leq a_{2}
\end{array}
$$

where $a_{1}, a_{2}$ are two arbitrary real numbers, and the maximization is over all jointly distributed random variables. Quite different from (30), the optimization problem (31) does not always have a Gaussian optimal solution.

Consider the special case where $a_{1}=a_{2}$. Denote by $\left(X_{1 G}^{*}, X_{2 G}^{*}\right)$ the optimal Gaussian solution of the optimization problem (31). We have $X_{1 G}^{*}=X_{2 G}^{*}$ almost surely, i.e., $X_{1 G}^{*}$ and $X_{2 G}^{*}$ must be aligned and have the same marginal distribution. Consider all jointly distributed $\left(X_{1}, X_{2}\right)$ where $X_{1}$ and $X_{2}$ have the same marginal density function $f$ which satisfies:

1. $h\left(X_{1}\right)=h\left(X_{1 G}^{*}\right)$;

2. $f$ is not log-concave.

By the result of Cover and Zhang [11], among all $\left(X_{1}, X_{2}\right)$ satisfying the above two conditions, there exists at least one that satisfies

$$
h\left(X_{1}+X_{2}\right)>h\left(2 X_{1}\right)=h\left(2 X_{1 G}^{*}\right)=h\left(X_{1 G}^{*}+X_{2 G}^{*}\right)
$$

where the inequality is strict. We thus conclude that, at least for this case, the optimization problem (31) does not have a Gaussian optimal solution.

Somewhat between the optimization problems (30) and (31) lies the following optimization problem:

$$
\begin{array}{ll}
\max _{X_{1}, X_{2}} & h\left(X_{1}+X_{2}\right) \\
\text { subject to } & \operatorname{Var}\left(X_{1}\right) \leq a_{1}, \quad h\left(X_{2}\right) \leq a_{2}
\end{array}
$$


where $a_{1}, a_{2}$ are two arbitrary real numbers with $a_{1} \geq 0$, and the maximization is over all jointly distributed random variables. To our best knowledge, whether this problem has a Gaussian optimal solution remains an open problem. However, we may use Corollary 15 to prove the following result along the line; the proof is in Appendix $G$.

Corollary 6 Let $Z$ be a Gaussian variable with strictly positive covariance. The optimization problem

$$
\begin{array}{ll}
\max _{X_{1}, X_{2}} & h\left(X_{1}+X_{2}+Z\right) \\
\text { subject to } & \operatorname{Var}\left(X_{1}\right) \leq a_{1}, \quad h\left(X_{2}+Z\right) \leq a_{2}
\end{array}
$$

where the maximization is over all jointly distributed random variables independent of $Z$ has a Gaussian optimal solution for all $a_{1} \geq 0$ and all $h(Z) \leq a_{2} \leq a_{2}^{*}$ where

$$
a_{2}^{*}=\frac{1}{2} \log _{e}\left(\pi e\left(a_{1}+4 \operatorname{Var}(Z)-\sqrt{a_{1}\left(a_{1}+4 \operatorname{Var}(Z)\right)}\right)\right) .
$$

\section{Concluding Remarks}

Information theoretic inequalities are important in proving the converse coding theorems for multiterminal source/channel coding problems. Motivated by the vector Gaussian broadcast channel and the distributed source coding with a single quadratic distortion constraint problem, we proved a new extremal inequality regarding the differential entropy of random vectors. As a corollary, this inequality yields a generalization of the classical vector EPI. As another corollary, this inequality sheds insight into maximizing the differential entropy of the sum of two jointly distributed random variables.

In this paper, we gave two proofs to the main result: one uses the enhancing idea of Weingarten, Steinberg and Shamai [9, and the other follows from the more traditional perturbation approach for proving EPI type of results. The perturbation proof approach allows us to uncover a new lemma on the monotonicity of Fisher information with regard to the Gaussian component of the involving random vector.

Finally, a few comments on the worst additive noise lemma (Lemma 8) are in order. A somewhat surprising fact about this result is that, even though it involves optimizing the entropy of the sum of two independent random vectors, the proof does not require EPI (but is only through a much less involved argument). In fact, except for the special case where $\mathbf{K}_{X}$ is proportional to $\mathbf{K}_{Z}$, the worst additive noise lemma cannot be proved by directly using the classical EPI.

In the literature, this seems to have caused some confusion between the classical vector EPI and the worst additive noise lemma, in that some applications of the classical vector 
EPI can actually be replaced by the relatively weaker worst additive noise lemma. Realizing the difference between these two results may help to generalize some of the existing results to the vector case without having to strengthen the classical vector EPI. See [16] for an example on how to use this observation to generalize Ozarow's solution of the scalar Gaussian twodescription problem [6] to the vector case.

\section{A Proof of the Right-Hand Sides of (15) as an Outer Bound}

We now prove that, without assuming that $\left(U, \widehat{\mathbf{Y}}_{1}\right)$ is jointly Gaussian with $\left(\mathbf{Y}_{1}, \mathbf{Y}_{2}\right)$, the right-hand sides of (15) are an outer bound for the rate region of the distributed source coding with a single quadratic distortion constraint problem. Note that $\mathbf{Y}_{1}$ and $\mathbf{Y}_{2}$ are jointly Gaussian. Without loss of generality, we may write

$$
\mathbf{Y}_{1}=\mathrm{AY}_{2}+\mathrm{Z}
$$

where $\mathbf{A}$ is an invertible matrix and $\mathbf{Z}$ is Gaussian and independent of $\mathbf{Y}_{2}$. Since there is no distortion constraint on $\mathbf{Y}_{2}$, we can always assume that $\mathbf{Y}_{1}$ is a degraded version of $\mathbf{Y}_{2}$ by relabelling $\mathbf{A} \mathbf{Y}_{2}$ as $\mathbf{Y}_{2}$. In this situation, an outer bound can be obtained similarly to that for the discrete memoryless degraded broadcast channel [17].

Let $W_{1}$ and $W_{2}$ be the encoded messages for $\left\{\mathbf{Y}_{1}[k]\right\}_{k=1}^{n}$ and $\left\{\mathbf{Y}_{2}[k]\right\}_{k=1}^{n}$, respectively. Denote $\underline{\mathbf{Y}_{i}^{k}}:=\left(\mathbf{Y}_{i}[1], \cdots, \mathbf{Y}_{i}[k]\right)$ and $U[k]:=\left(W_{2}, \underline{\mathbf{Y}_{1}^{k-1}}\right)$. We have

$$
\begin{aligned}
n R_{2} & =H\left(W_{2}\right) \\
& \geq H\left(W_{2}\right)-H\left(W_{2} \mid \underline{\mathbf{Y}_{2}^{n}}\right) \\
& =I\left(W_{2} ; \underline{\mathbf{Y}_{2}^{n}}\right) \\
& =\sum_{k=1}^{n} I\left(W_{2} ; \mathbf{Y}_{2}[k] \mid \underline{\mathbf{Y}_{2}^{k-1}}\right) \\
& =\sum_{k=1}^{n}\left(h\left(\mathbf{Y}_{2}[k] \mid \underline{\mathbf{Y}_{2}^{k-1}}\right)-h\left(\mathbf{Y}_{2}[k] \mid W_{2}, \underline{\mathbf{Y}_{2}^{k-1}}\right)\right) \\
& =\sum_{k=1}^{n}\left(h\left(\mathbf{Y}_{2}[k]\right)-h\left(\mathbf{Y}_{2}[k] \mid W_{2}, \underline{\mathbf{Y}_{2}^{k-1}}, \underline{\mathbf{Y}_{1}^{k-1}}\right)\right) \\
& \geq \sum_{k=1}^{n}\left(h\left(\mathbf{Y}_{2}[k]\right)-h\left(\mathbf{Y}_{2}[k] \mid W_{2}, \underline{\mathbf{Y}_{1}^{k-1}}\right)\right) \\
& =\sum_{k=1}^{n} I\left(U[k] ; \mathbf{Y}_{2}[k]\right)
\end{aligned}
$$


where (42) follows from the fact that $\underline{Y}_{1}^{k-1}$ is a degraded version of $\underline{Y}_{2}^{k-1}$ for $k=1, \cdots, n$. Furthermore,

$$
\begin{aligned}
n R_{1} & =H\left(W_{1}\right) \\
& \geq H\left(W_{1}\right)-H\left(W_{1} \mid W_{2}, \underline{\mathbf{Y}_{1}^{n}}\right) \\
& =I\left(W_{1} ; W_{2}, \underline{\mathbf{Y}_{1}^{n}}\right) \\
& =I\left(W_{1} ; \underline{\mathbf{Y}_{1}^{n} \mid} \mid W_{2}\right)+I\left(W_{1} ; W_{2}\right) \\
& \geq I\left(W_{1} ; \underline{\mathbf{Y}_{1}^{n}} \mid W_{2}\right) \\
& =\sum_{k=1}^{n} I\left(W_{1} ; \mathbf{Y}_{1}[k] \mid W_{2}, \underline{\mathbf{Y}_{1}^{k-1}}\right) \\
\geq & \sum_{k=1}^{n} I\left(\widehat{\mathbf{Y}}_{1}[k] ; \mathbf{Y}_{1}[k] \mid W_{2}, \underline{\mathbf{Y}_{1}^{k-1}}\right) \\
& =\sum_{k=1}^{n} I\left(\widehat{\mathbf{Y}}_{1}[k] ; \mathbf{Y}_{1}[k] \mid U[k]\right)
\end{aligned}
$$

where (51) follows from the Markov chain $\mathbf{Y}_{1}[k] \leftrightarrow\left(W_{1}, W_{2}\right) \leftrightarrow \widehat{\mathbf{Y}}_{1}[k]$ for $k=1, \cdots, n$. Finally, let $Q$ be a random variable uniformly distributed over $\{1, \cdots, n\}$ and independent of any other random variables/vectors. The desired outer bound (15) is proved if we define

$$
U:=(Q, U[Q]), \quad \widehat{\mathbf{Y}}_{1}:=\widehat{\mathbf{Y}}_{1}[Q], \quad \mathbf{Y}_{1}:=\mathbf{Y}_{1}[Q], \quad \mathbf{Y}_{2}:=\mathbf{Y}_{2}[Q]
$$

\section{B A Direct Proof of Theorem 1}

To simplify the notation, let us denote the optimization problem (8) by $P$ and the optimal value of $P$ by $(P)$. To show that $\mathrm{P}$ has a Gaussian optimal solution, it is sufficient to show that $(\mathrm{P})=\left(P_{G}\right)$ where $P_{G}$ is the Gaussian version of $P$ by restricting the solution space within Gaussian distributions. Since restricting the solution space can only decrease the value of a maximization problem, we readily have $(P) \geq\left(P_{G}\right)$.

To prove the reverse inequality $(P) \leq\left(P_{G}\right)$, we shall consider an auxiliary optimization problem $\widetilde{P}$ and its Gaussian version $\widetilde{P}_{G}$. Specifically, if we can find an optimization problem $\widetilde{P}$ such that

$$
(P) \leq(\widetilde{P}), \quad(\widetilde{P})=\left(\widetilde{P}_{G}\right), \quad\left(\widetilde{P}_{G}\right)=\left(P_{G}\right)
$$

we will have $(P) \leq\left(P_{G}\right)$, and the desired result $(P)=\left(P_{G}\right)$ will follow.

Let $\mathbf{X}_{G}^{*}$ be an optimal solution of $P_{G}$ and let $\mathbf{K}_{X}^{*}$ be the covariance matrix of $\mathbf{X}_{G}^{*}$. Then 
$\mathbf{K}_{X}^{*}$ is an optimal solution to the conic program:

$$
\begin{array}{ll}
\max _{\mathbf{K}_{X}} & (1 / 2) \log _{e}\left|\mathbf{K}_{X}+\mathbf{K}_{Z_{1}}\right|-(\mu / 2) \log _{e}\left|\mathbf{K}_{X}+\mathbf{K}_{Z_{2}}\right| \\
\text { subject to } & \mathbf{0} \preceq \mathbf{K}_{X} \preceq \mathbf{S}
\end{array}
$$

where the maximization is over all real symmetric matrices satisfying the constraints. As shown in [9], $\mathbf{K}_{X}^{*}$ must satisfy the following KKT-like necessary conditions:

$$
\begin{aligned}
(1 / 2)\left(\mathbf{K}_{X}^{*}+\mathbf{K}_{Z_{1}}\right)^{-1}+\mathbf{M}_{1} & =(\mu / 2)\left(\mathbf{K}_{X}^{*}+\mathbf{K}_{Z_{2}}\right)^{-1}+\mathbf{M}_{2}, \\
\mathbf{M}_{1} \mathbf{K}_{X}^{*} & =\mathbf{0} \\
\mathbf{M}_{2}\left(\mathbf{S}-\mathbf{K}_{X}^{*}\right) & =\mathbf{0}
\end{aligned}
$$

where $\mathbf{M}_{1}, \mathbf{M}_{2} \succeq \mathbf{0}$ are Lagrange multipliers corresponding to the constraints $\mathbf{K}_{X} \succeq \mathbf{0}$ and $\mathbf{K}_{X} \preceq \mathbf{S}$, respectively. Let $\mathbf{K}_{\widetilde{Z}_{1}}$ and $\mathbf{K}_{\widetilde{Z}_{2}}$ be two real symmetric matrices satisfying:

$$
\begin{aligned}
(1 / 2)\left(\mathbf{K}_{X}^{*}+\mathbf{K}_{Z_{1}}\right)^{-1}+\mathbf{M}_{1} & =(1 / 2)\left(\mathbf{K}_{X}^{*}+\mathbf{K}_{\widetilde{Z}_{1}}\right)^{-1}, \\
(\mu / 2)\left(\mathbf{K}_{X}^{*}+\mathbf{K}_{Z_{2}}\right)^{-1}+\mathbf{M}_{2} & =(\mu / 2)\left(\mathbf{K}_{X}^{*}+\mathbf{K}_{\widetilde{Z}_{2}}\right)^{-1} .
\end{aligned}
$$

We have the following lemma, which was proved in [9].

Lemma 7 For $\mathbf{K}_{X}^{*}, \mathbf{K}_{Z_{i}}, \mathbf{K}_{\widetilde{Z}_{i}}, \mathbf{M}_{i}, i=1,2$, related through (56) to (60) and $\mu \geq 1$, we have $\mathbf{0} \preceq \mathbf{K}_{\widetilde{Z}_{1}} \preceq \mathbf{K}_{Z_{1}}$ and $\mathbf{K}_{\widetilde{Z}_{1}} \preceq \mathbf{K}_{\widetilde{Z}_{2}} \preceq \mathbf{K}_{Z_{2}}$.

Let $\widetilde{\mathbf{Z}}_{1}$ and $\widetilde{\mathbf{Z}}_{2}$ be two Gaussian vectors with covariance matrix $\mathbf{K}_{\widetilde{Z}_{1}}$ and $\mathbf{K}_{\widetilde{Z}_{2}}$, respectively. By Lemma $\mathbf{Z}$ both $\mathbf{K}_{\widetilde{Z}_{1}}$ and $\mathbf{K}_{\widetilde{Z}_{2}}$ are positive semidefinite and hence both may serve as covariance matrices of random vectors. We now define the auxiliary optimization problem $\widetilde{P}$ as:

$$
\begin{array}{ll}
\max _{\mathbf{X}} & h\left(\mathbf{X}+\widetilde{\mathbf{Z}}_{1}\right)-\mu h\left(\mathbf{X}+\widetilde{\mathbf{Z}}_{2}\right)+c \\
\text { subject to } & \operatorname{Cov}(\mathbf{X}) \preceq \mathbf{S}
\end{array}
$$

where the constant $c$ is defined as

$$
c:=h\left(\mathbf{Z}_{1}\right)-h\left(\widetilde{\mathbf{Z}}_{1}\right)+\mu\left(h\left(\mathbf{X}_{S}+\widetilde{\mathbf{Z}}_{2}\right)-h\left(\mathbf{X}_{S}+\mathbf{Z}_{2}\right)\right)
$$

$\mathbf{X}_{S}$ is Gaussian vector with covariance matrix $\mathbf{S}$ and independent of $\mathbf{Z}_{2}$ and $\widetilde{\mathbf{Z}}_{2}$, and the maximization is over all random vectors independent of $\widetilde{\mathbf{Z}}_{1}$ and $\widetilde{\mathbf{Z}}_{2} \cdot{ }^{4}$ Next, we show that the auxiliary optimization problem $\widetilde{P}$ so defined satisfies the desired chain of relationships (54).

Step 1: Proving $(P) \leq(\widetilde{P})$. Since the optimization problems $\mathrm{P}$ and $\widetilde{\mathrm{P}}$ have the same solution space, to show $(P) \leq(\widetilde{P})$, it is sufficient to show that, for each admissible solution, the value of the objective function of $P$ is less or equal than that of $\widetilde{P}$.

\footnotetext{
${ }^{4}$ In [9], the process of replacing $\mathbf{Z}_{1}$ and $\mathbf{Z}_{2}$ by $\widetilde{\mathbf{Z}}_{1}$ and $\widetilde{\mathbf{Z}}_{2}$, respectively is called enhancing.
} 
The difference between the objective functions of $\mathrm{P}$ and $\widetilde{\mathrm{P}}$ can be written as:

$$
\begin{aligned}
h\left(\mathbf{X}+\mathbf{Z}_{1}\right)-h\left(\mathbf{Z}_{1}\right)-h\left(\mathbf{X}+\widetilde{\mathbf{Z}}_{1}\right)+h\left(\widetilde{\mathbf{Z}}_{1}\right) & \\
& -\mu\left(h\left(\mathbf{X}+\mathbf{Z}_{2}\right)-h\left(\mathbf{X}+\widetilde{\mathbf{Z}}_{2}\right)-h\left(\mathbf{X}_{S}+\mathbf{Z}_{2}\right)+h\left(\mathbf{X}_{S}+\widetilde{\mathbf{Z}}_{2}\right)\right) .
\end{aligned}
$$

By Lemma [7] we have $\mathbf{K}_{Z_{i}} \succeq \mathbf{K}_{\widetilde{Z}_{i}}$ for $i=1,2$. Therefore, we may write $\mathbf{Z}_{i}=\widetilde{\mathbf{Z}}_{i}+\widehat{\mathbf{Z}}_{i}$ where $\widehat{\mathbf{Z}}_{i}$ is a Gaussian vector independent of $\widetilde{\mathbf{Z}}_{i}$. We thus have

$$
\begin{aligned}
h\left(\mathbf{X}+\mathbf{Z}_{1}\right)-h\left(\mathbf{Z}_{1}\right)-h\left(\mathbf{X}+\widetilde{\mathbf{Z}}_{1}\right)+h\left(\widetilde{\mathbf{Z}}_{1}\right) & =I\left(\mathbf{X} ; \mathbf{X}+\mathbf{Z}_{1}\right)-I\left(\mathbf{X} ; \mathbf{X}+\widetilde{\mathbf{Z}}_{1}\right) \\
& =I\left(\mathbf{X} ; \mathbf{X}+\widetilde{\mathbf{Z}}_{1}+\widehat{\mathbf{Z}}_{1}\right)-I\left(\mathbf{X} ; \mathbf{X}+\widetilde{\mathbf{Z}}_{1}\right) \\
& \leq 0
\end{aligned}
$$

where the inequality is due to the Markov chain $\mathbf{X} \leftrightarrow \mathbf{X}+\widetilde{\mathbf{Z}}_{1} \leftrightarrow \mathbf{X}+\widetilde{\mathbf{Z}}_{1}+\widehat{\mathbf{Z}}_{1}$. Furthermore,

$$
\begin{aligned}
h(\mathbf{X} & \left.+\mathbf{Z}_{2}\right)-h\left(\mathbf{X}+\widetilde{\mathbf{Z}}_{2}\right)-h\left(\mathbf{X}_{S}+\mathbf{Z}_{2}\right)+h\left(\mathbf{X}_{S}+\widetilde{\mathbf{Z}}_{2}\right) \\
& =h\left(\mathbf{X}+\widetilde{\mathbf{Z}}_{2}+\widehat{\mathbf{Z}}_{2}\right)-h\left(\mathbf{X}+\widetilde{\mathbf{Z}}_{2}\right)-h\left(\mathbf{X}_{S}+\widetilde{\mathbf{Z}}_{2}+\widehat{\mathbf{Z}}_{2}\right)+h\left(\mathbf{X}_{S}+\widetilde{\mathbf{Z}}_{2}\right) \\
& =I\left(\widehat{\mathbf{Z}}_{2} ; \mathbf{X}+\widetilde{\mathbf{Z}}_{2}+\widehat{\mathbf{Z}}_{2}\right)-I\left(\widehat{\mathbf{Z}}_{2} ; \mathbf{X}_{S}+\widetilde{\mathbf{Z}}_{2}+\widehat{\mathbf{Z}}_{2}\right) .
\end{aligned}
$$

To bound from below the right-hand side of (68), we need the following worst additive noise lemma proved by Ihara [12] and by Diggavi and Cover [13, Lemma II.2].

Lemma $\mathbf{8}$ Let $\mathbf{Z}$ be a Gaussian vector with covariance matrix $\mathbf{K}_{Z}$ and let $\mathbf{K}_{X}$ be a positive semidefinite matrix. The optimization problem

$$
\begin{array}{ll}
\max _{\mathbf{X}} & h(\mathbf{X})-h(\mathbf{X}+\mathbf{Z}) \\
\text { subject to } & \operatorname{Cov}(\mathbf{X})=\mathbf{K}_{X}
\end{array}
$$

where the maximization is over all random vectors independent of $\mathbf{Z}$ has a Gaussian optimal solution, no matter $\mathbf{K}_{X}$ and $\mathbf{K}_{Z}$ are proportional or not.

Remark: Note that the objective function of the optimization problem (69) is equal to $-I(\mathbf{Z} ; \mathbf{Z}+\mathbf{X})$. Consider $\mathbf{Z}$ as the channel input and $\mathbf{Z}+\mathbf{X}$ the channel output. Lemma 8 simply states that given a Gaussian input, the Gaussian additive noise channel is a worst one among all additive noise channels with a fixed noise covariance matrix.

Let $\mathbf{X}_{G}$ be a Gaussian vector with the same covariance matrix as that of $\mathbf{X}$ and independent of $\widetilde{\mathbf{Z}}_{2}$ and $\widehat{\mathbf{Z}}_{2}$. By Lemma 8 , we have

$$
I\left(\widehat{\mathbf{Z}}_{2} ; \mathbf{X}+\widetilde{\mathbf{Z}}_{2}+\widehat{\mathbf{Z}}_{2}\right)-I\left(\widehat{\mathbf{Z}}_{2} ; \mathbf{X}_{G}+\widetilde{\mathbf{Z}}_{2}+\widehat{\mathbf{Z}}_{2}\right) \geq 0 .
$$

Since $\mathbf{X}_{G}$ is Gaussian with covariance matrix $\operatorname{Cov}\left(\mathbf{X}_{G}\right)=\operatorname{Cov}(\mathbf{X}) \preceq \mathbf{S}=\operatorname{Cov}\left(\mathbf{X}_{S}\right)$, we may write $\mathbf{X}_{S}=\mathbf{X}_{G}+\widehat{\mathbf{X}}_{G}$ where $\widehat{\mathbf{X}}_{G}$ is a Gaussian vector independent of $\mathbf{X}_{G}$. We have

$$
\begin{aligned}
& I\left(\widehat{\mathbf{Z}}_{2} ; \mathbf{X}_{G}+\widetilde{\mathbf{Z}}_{2}+\widehat{\mathbf{Z}}_{2}\right)-I\left(\widehat{\mathbf{Z}}_{2} ; \mathbf{X}_{S}+\widetilde{\mathbf{Z}}_{2}+\widehat{\mathbf{Z}}_{2}\right) \\
& \quad=I\left(\widehat{\mathbf{Z}}_{2} ; \mathbf{X}_{G}+\widetilde{\mathbf{Z}}_{2}+\widehat{\mathbf{Z}}_{2}\right)-I\left(\widehat{\mathbf{Z}}_{2} ; \widehat{\mathbf{X}}_{G}+\mathbf{X}_{G}+\widetilde{\mathbf{Z}}_{2}+\widehat{\mathbf{Z}}_{2}\right) \\
& \quad \geq 0
\end{aligned}
$$


where the inequality follows from the Markov chain $\widehat{\mathbf{Z}}_{2} \leftrightarrow \mathbf{X}_{G}+\widetilde{\mathbf{Z}}_{2}+\widehat{\mathbf{Z}}_{2} \leftrightarrow \widehat{\mathbf{X}}_{G}+\mathbf{X}_{G}+\widetilde{\mathbf{Z}}_{2}+\widehat{\mathbf{Z}}_{2}$. Putting (170) and (72) together, we obtain from (68)

$$
h\left(\mathbf{X}+\mathbf{Z}_{2}\right)-h\left(\mathbf{X}+\widetilde{\mathbf{Z}}_{2}\right)-h\left(\mathbf{X}_{S}+\mathbf{Z}_{2}\right)+h\left(\mathbf{X}_{S}+\widetilde{\mathbf{Z}}_{2}\right) \geq 0
$$

Substituting (66) and (73) into (63), we conclude that $(P) \leq(\widetilde{P})$ for all $\mu \geq 1$.

Step 2: Proving $(\widetilde{P})=\left(\widetilde{P}_{G}\right)$. To show that $(\widetilde{P})=\left(\widetilde{P}_{G}\right)$, it is sufficient to show that $\mathbf{X}_{G}^{*}$, the optimal solution of $P_{G}$ characterized in Step 1, is also an optimal solution of $\widetilde{P}$. Since $\mathbf{X}_{G}^{*}$ is by construction Gaussian, this will lead to the conclusion $(\widetilde{P})=\left(\widetilde{P}_{G}\right)$.

Let us first assume $\mu>1$. By Lemma [7, we have $\mathbf{K}_{\widetilde{Z}_{2}} \succeq \mathbf{K}_{\widetilde{Z}_{1}}$. Therefore, we may write $\widetilde{\mathbf{Z}}_{2}=\widetilde{\mathbf{Z}}_{1}+\widetilde{\mathbf{Z}}$ where $\widetilde{\mathbf{Z}}$ is a Gaussian vector with covariance matrix $\mathbf{K}_{\widetilde{Z}}=\mathbf{K}_{\widetilde{Z}_{2}}-\mathbf{K}_{\widetilde{Z}_{1}}$ and independent of $\widetilde{\mathbf{Z}}_{1}$. It follows that for any random vector $\mathbf{X}$,

$$
\begin{aligned}
h(\mathbf{X} & \left.+\widetilde{\mathbf{Z}}_{1}\right)-\mu h\left(\mathbf{X}+\widetilde{\mathbf{Z}}_{2}\right) \\
& =h\left(\mathbf{X}+\widetilde{\mathbf{Z}}_{1}\right)-\mu h\left(\mathbf{X}+\widetilde{\mathbf{Z}}_{1}+\widetilde{\mathbf{Z}}\right) \\
& \leq h\left(\mathbf{X}+\widetilde{\mathbf{Z}}_{1}\right)-\frac{\mu n}{2} \log _{e}\left(e^{2 h\left(\mathbf{X}+\widetilde{\mathbf{Z}}_{1}\right) / n}+e^{2 h(\widetilde{\mathbf{Z}}) / n}\right) \\
& \leq h(\widetilde{\mathbf{Z}})-(n / 2)(\mu-1)-(\mu n / 2) \log _{e}\left(e^{2\left(h(\widetilde{\mathbf{Z}})-\frac{n}{2}(\mu-1)\right) / n}+e^{2 h(\widetilde{\mathbf{Z}}) / n}\right) \\
& =(n / 2)\left((\mu-1) \log _{e}(\mu-1)-\mu \log _{e} \mu\right)-(\mu-1) h(\widetilde{\mathbf{Z}}) .
\end{aligned}
$$

where (75) follows from the classical vector EPI, and (76) follows from the fact that for $\mu>1$, the function $f$ defined in (5) has a global maxima at (6).

Next, we verify that the upper bound on the right-hand side of (77) can be achieved by $\mathbf{X}_{G}^{*}$. Substituting (59) and (60) into the KKT-like condition (56), we obtain

$$
\left(\mathbf{K}_{X}^{*}+\mathbf{K}_{\widetilde{Z}_{1}}\right)^{-1}=\mu\left(\mathbf{K}_{X}^{*}+\mathbf{K}_{\widetilde{Z}_{2}}\right)^{-1} .
$$

We have

$$
\mathbf{K}_{X}^{*}+\mathbf{K}_{\widetilde{Z}_{1}}=(\mu-1)^{-1} \mathbf{K}_{\widetilde{Z}}, \quad \mathbf{K}_{X}^{*}+\mathbf{K}_{\widetilde{Z}_{2}}=\mu(\mu-1)^{-1} \mathbf{K}_{\widetilde{Z}}
$$

and hence

$h\left(\mathbf{X}_{G}^{*}+\widetilde{\mathbf{Z}}_{1}\right)=h(\widetilde{\mathbf{Z}})-(n / 2) \log _{e}(\mu-1), \quad h\left(\mathbf{X}_{G}^{*}+\widetilde{\mathbf{Z}}_{2}\right)=h(\widetilde{\mathbf{Z}})-(n / 2)\left(\log _{e}(\mu-1)-\log _{e} \mu\right)$.

This gives

$$
h\left(\mathbf{X}_{G}^{*}+\widetilde{\mathbf{Z}}_{1}\right)-\mu h\left(\mathbf{X}_{G}^{*}+\widetilde{\mathbf{Z}}_{2}\right)=(n / 2)\left((\mu-1) \log _{e}(\mu-1)-\mu \log _{e} \mu\right)-(\mu-1) h(\widetilde{\mathbf{Z}}) .
$$

Substituting (81) into the right-hand side of (77), we obtain

$$
h\left(\mathbf{X}+\widetilde{\mathbf{Z}}_{1}\right)-\mu h\left(\mathbf{X}+\widetilde{\mathbf{Z}}_{2}\right) \leq h\left(\mathbf{X}_{G}^{*}+\widetilde{\mathbf{Z}}_{1}\right)-\mu h\left(\mathbf{X}_{G}^{*}+\widetilde{\mathbf{Z}}_{2}\right) .
$$


We thus conclude that $\mathbf{X}_{G}^{*}$ is a Gaussian optimal solution of $\widetilde{P}$ for all $\mu>1$.

For $\mu=1$, by (178) we have $\mathbf{K}_{\widetilde{Z}_{1}}=\mathbf{K}_{\widetilde{Z}_{2}}$. Therefore in this case, the objective function of $\widetilde{P}$ is a constant and $\mathbf{X}_{G}^{*}$ is a trivial Gaussian optimal solution.

Step 3: Proving $\left(\widetilde{P}_{G}\right)=\left(P_{G}\right)$. Since $\mathbf{X}_{G}^{*}$ is an optimal solution of both $\widetilde{P}_{G}$ and $P_{G}$, to show $\left(\widetilde{P}_{G}\right)=\left(P_{G}\right)$, we only need to compare the objective functions of $\widetilde{P}_{G}$ and $P_{G}$ evaluated at $\mathbf{X}_{G}^{*}$. The following lemma, proved in [9], shows that the objective functions of $\widetilde{P}_{G}$ and $P_{G}$ take equal values at $\mathbf{X}_{G}^{*}$ and thus proves the desired result $\left(\widetilde{P}_{G}\right)=\left(P_{G}\right)$.

Lemma $\mathbf{9}$ For $\mathbf{K}_{X}^{*}, \mathbf{K}_{Z_{i}}, \mathbf{K}_{\widetilde{Z}_{i}}, \mathbf{M}_{i}, i=1,2$, defined through (56) to (60) and $\mu \geq 1$, we have

$$
\begin{aligned}
\left(\mathbf{K}_{X}^{*}+\mathbf{K}_{\widetilde{Z}_{1}}\right)^{-1} \mathbf{K}_{Z_{1}} & =\left(\mathbf{K}_{X}^{*}+\mathbf{K}_{Z_{1}}\right)^{-1} \mathbf{K}_{\widetilde{Z}_{1}} \\
\left(\mathbf{K}_{X}^{*}+\mathbf{K}_{\widetilde{Z}_{2}}\right)^{-1}\left(\mathbf{S}+\mathbf{K}_{\widetilde{Z}_{2}}\right) & =\left(\mathbf{K}_{X}^{*}+\mathbf{K}_{Z_{2}}\right)^{-1}\left(\mathbf{S}+\mathbf{K}_{Z_{2}}\right) .
\end{aligned}
$$

Combining Steps 1-3, we conclude that the optimization problem (8) has a Gaussian optimal solution for all $\mu \geq 1$. This completes a direct proof of Theorem 1 using the classical vector EPI.

Remark: It should now be clear why we need the auxiliary optimization problem $\widetilde{P}$ : to directly apply the classical vector EPI to solve the optimization problem $P$, we need $\mathbf{K}_{X}^{*}+\mathbf{K}_{Z_{1}}$ and $\mathbf{K}_{X}^{*}+\mathbf{K}_{Z_{2}}$ to be proportional. However, a quick look at the KKT-like conditions (566) reveals that to guarantee the required proportionality, we need both Lagrange multipliers $\mathbf{M}_{1}$ and $\mathbf{M}_{2}$ to be zero. Thus, the purpose of enhancing is to absorb the possibly nonzero Lagrange multipliers $\mathbf{M}_{1}, \mathbf{M}_{2}$ into the covariance matrices of $\mathbf{Z}_{1}$ and $\mathbf{Z}_{2}$, respectively, creating a new optimization problem that can be directly solved by the classical vector EPI. The choice of the constant $c$ in $\widetilde{P}$ was motivated by the vector Gaussian broadcast channel problem.

\section{A Perturbation Proof of Theorem 1}

To show that the optimization problem (8) has a Gaussian optimal solution, it is sufficient to show that for any random vector $\mathbf{X}_{0}$ with $\operatorname{Cov}\left(\mathbf{X}_{0}\right) \preceq \mathbf{S}$, there exists an admissible Gaussian solution at which the objective function is greater or equal than that at $\mathbf{X}_{0}$. In particular, we may choose the Gaussian solution to be an optimal Gaussian solution to the optimization problem:

$$
\begin{cases}\max _{\mathbf{X}} & h\left(\mathbf{X}+\mathbf{Z}_{1}\right)-\mu h\left(\mathbf{X}+\mathbf{Z}_{2}\right) \\ \text { subject to } & \operatorname{Cov}(\mathbf{X}) \preceq \operatorname{Cov}\left(\mathbf{X}_{0}\right)\end{cases}
$$




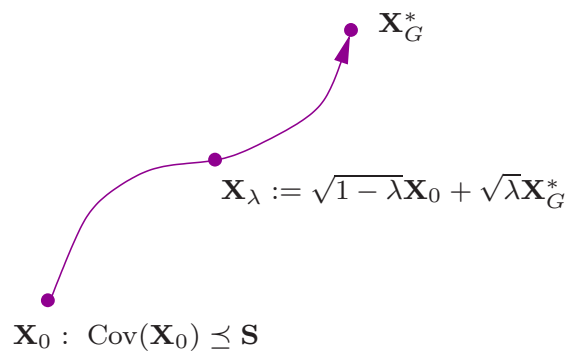

Figure 1: A monotone path connecting $\mathbf{X}_{0}$ and $\mathbf{X}_{G}^{*}$.

where the maximization is over all random vectors independent of $\mathbf{Z}_{1}$ and $\mathbf{Z}_{2}$. Without loss of generality, we may assume $\operatorname{Cov}\left(\mathbf{X}_{0}\right)=\mathbf{S}$. Under this assumption, the optimization problem (85) coincides with (86) and the optimal Gaussian solution of (85) becomes $\mathbf{X}_{G}^{*}$. Other notations developed in the basic proof of Theorem 1 can be reused as well.

The following lemma proves, for a special case, the desired result:

$$
h\left(\mathbf{X}_{0}+\mathbf{Z}_{1}\right)-\mu h\left(\mathbf{X}_{0}+\mathbf{Z}_{2}\right) \leq h\left(\mathbf{X}_{G}^{*}+\mathbf{Z}_{1}\right)-\mu h\left(\mathbf{X}_{G}^{*}+\mathbf{Z}_{2}\right)
$$

for any random vector $\mathbf{X}_{0}$ with covariance matrix $\mathbf{S}$ and any $\mu \geq 1$. The proof of the lemma is deferred to Appendix $\mathrm{E}$

Lemma 10 Let $\mathbf{X}_{G}^{*}$ be an optimal Gaussian solution of the optimization problem (8). For any random vector $\mathbf{X}_{0}$ with covariance matrix $\mathbf{S}$ and any $0 \leq \lambda \leq 1$, let

$$
\mathbf{X}_{\lambda}:=\sqrt{1-\lambda} \mathbf{X}_{0}+\sqrt{\lambda} \mathbf{X}_{G}^{*}
$$

If the Lagrange multiplier $\mathbf{M}_{1}$ in the KKT-like condition (56) equals zero, then

$$
g(\lambda):=h\left(\mathbf{X}_{\lambda}+\mathbf{Z}_{1}\right)-\mu h\left(\mathbf{X}_{\lambda}+\mathbf{Z}_{2}\right)
$$

is a monotone increasing function of $\lambda$ for $0 \leq \lambda \leq 1$ and $\mu \geq 1$.

By the definition of $g(\lambda)$ in (88), the desired result (86) can be written as $g(0) \leq g(1)$, which follows easily from the monotonicity of $g(\lambda)$ for $0 \leq \lambda \leq 1$ of Lemma [10. A depiction of Lemma 10 is shown in Figure 1].

For the general case where $\mathbf{M}_{1}$ is nonzero, we may consider first to enhance $\mathbf{Z}_{1}$ to create a new optimization problem in which the corresponding $\mathbf{M}_{1}$ equals zero. We may then apply Lemma 10 to the new problem to obtain the desired result [86). Formally, this can be done as follows. Define

$$
g(\mathbf{X}):=h\left(\mathbf{X}+\mathbf{Z}_{1}\right)-\mu h\left(\mathbf{X}+\mathbf{Z}_{2}\right)-h\left(\mathbf{Z}_{1}\right)
$$


Using the definition of $g(\mathbf{X})$, the desired result (86) can be written as $g\left(\mathbf{X}_{0}\right) \leq g\left(\mathbf{X}_{G}^{*}\right)$. To show $g\left(\mathbf{X}_{0}\right) \leq g\left(\mathbf{X}_{G}^{*}\right)$, we consider an auxiliary function $\widetilde{g}(\mathbf{X})$ defined as

$$
\widetilde{g}(\mathbf{X}):=h\left(\mathbf{X}+\widetilde{\mathbf{Z}}_{1}\right)-\mu h\left(\mathbf{X}+\mathbf{Z}_{2}\right)-h\left(\widetilde{\mathbf{Z}}_{1}\right)
$$

where $\widetilde{\mathbf{Z}}_{1}$ is a Gaussian vector with covariance matrix $\mathbf{K}_{\widetilde{Z}_{1}}$ which is defined in (59). If we can show

$$
g\left(\mathbf{X}_{0}\right) \leq \widetilde{g}\left(\mathbf{X}_{0}\right), \quad \widetilde{g}\left(\mathbf{X}_{0}\right) \leq \widetilde{g}\left(\mathbf{X}_{G}^{*}\right), \quad \widetilde{g}\left(\mathbf{X}_{G}^{*}\right)=g\left(\mathbf{X}_{G}^{*}\right),
$$

the desired inequality $g\left(\mathbf{X}_{0}\right) \leq g\left(\mathbf{X}_{G}^{*}\right)$ will follow.

Assume that $\mathbf{Z}_{2}$ and $\widetilde{\mathbf{Z}}_{2}$ have the same distribution. The statements $g\left(\mathbf{X}_{0}\right) \leq \widetilde{g}\left(\mathbf{X}_{0}\right)$ and $\widetilde{g}\left(\mathbf{X}_{G}^{*}\right)=g\left(\mathbf{X}_{G}^{*}\right)$ are equivalent to $(P) \leq(\widetilde{P})$ and $\left(\widetilde{P}_{G}\right)=\left(P_{G}\right)$, respectively. Since both $(P) \leq(\widetilde{P})$ and $\left(\widetilde{P}_{G}\right)=\left(P_{G}\right)$ have been shown in the basic proof of Theorem $\mathbf{1}$ all we need to show now is $\widetilde{g}\left(\mathbf{X}_{0}\right) \leq \widetilde{g}\left(\mathbf{X}_{G}^{*}\right)$. Consider the optimization problem

$$
\begin{cases}\max _{\mathbf{X}} & h\left(\mathbf{X}+\widetilde{\mathbf{Z}}_{1}\right)-\mu h\left(\mathbf{X}+\mathbf{Z}_{2}\right) \\ \text { subject to } & \operatorname{Cov}(\mathbf{X}) \preceq \mathbf{S}\end{cases}
$$

where the maximization is over all random vectors independent of $\widetilde{\mathbf{Z}}_{1}$ and $\mathbf{Z}_{2}$. By the definition of $\widetilde{\mathbf{Z}}_{1}$, the Lagrange multiplier in the optimization problem (92) that corresponds to $\mathbf{M}_{1}$ in the KKT-like condition (56) must equal zero. Applying Lemma 10, we obtain the desired result $\widetilde{g}\left(\mathbf{X}_{0}\right) \leq \widetilde{g}\left(\mathbf{X}_{G}^{*}\right)$. This completes the perturbation proof of Theorem $\mathbf{1}$

Remark: In the direct proof of Theorem $\mathbf{1}$ we enhance both $\mathbf{Z}_{1}$ and $\mathbf{Z}_{2}$ to obtain the desired proportionality so that the classical vector EPI can be used solve the problem. In the perturbation proof, however, we only need to enhance $\mathbf{Z}_{1}$ so that the monotone result of Lemma 10 may apply. Neither the classical EPI nor the worst additive noise result of Lemma 8 is needed in the perturbation proof of Theorem 1

\section{Proof of Lemma 2}

We first give some preliminaries on Fisher information and score function. The material can be found, for example, in [18, Chapter 3.2].

Definition 11 For a random vector $\mathbf{U}$ with a differentiable density function $f_{U}$ in $\mathcal{R}^{n}$, the Fisher information matrix $\mathbf{J}(\cdot)$ is defined as

$$
\mathbf{J}(\mathbf{U}):=\mathbb{E}\left[\boldsymbol{\rho}_{U}(\mathbf{U}) \boldsymbol{\rho}_{U}^{t}(\mathbf{U})\right]
$$


where the vector-valued score function

$$
\boldsymbol{\rho}_{U}(\mathbf{u}):=\nabla \log _{e} f_{U}(\mathbf{u})=\left(\frac{\partial}{\partial u_{1}} \log _{e} f_{U}(\mathbf{u}), \cdots, \frac{\partial}{\partial u_{n}} \log _{e} f_{U}(\mathbf{u})\right)^{t} .
$$

The following results on score function and Fisher information are known.

Lemma 12 The following statements on score function are true.

1. (Gaussian Distribution) If $\mathbf{U}$ is a Gaussian vector with zero mean and positive definite covariance matrix $\mathbf{K}_{U}$, then

$$
\rho_{U}(\mathbf{u})=-\mathbf{K}_{U}^{-1} \mathbf{u} .
$$

2. (Stein Identity) For any smooth scalar-valued function $f$ well behaved at infinity, we have

$$
\mathbb{E}\left[f(\mathbf{U}) \boldsymbol{\rho}_{U}(\mathbf{U})\right]=-\mathbb{E}[\nabla f(\mathbf{U})]
$$

In particular, we have

$$
\mathbb{E}\left[\mathbf{U} \boldsymbol{\rho}_{U}^{t}(\mathbf{U})\right]=-\mathbf{I}
$$

where $\mathbf{I}$ is the identity matrix.

3. (Behavior on Convolution) If $\mathbf{U}, \mathbf{V}$ are two independent random vectors and $\mathbf{W}=$ $\mathbf{U}+\mathbf{V}$, then

$$
\boldsymbol{\rho}_{W}(\mathbf{w})=\mathbb{E}\left[\boldsymbol{\rho}_{U}(\mathbf{U}) \mid \mathbf{W}=\mathbf{w}\right]=\mathbb{E}\left[\boldsymbol{\rho}_{V}(\mathbf{V}) \mid \mathbf{W}=\mathbf{w}\right] .
$$

Lemma 13 The following statements on Fisher information are true.

1. (Cramér-Rao Inequality) For any random vector $\mathbf{U}$ with covariance matrix $\mathbf{K}_{U}$, we have

$$
\mathbf{J}(\mathbf{U}) \succeq \mathbf{K}_{U}^{-1}
$$

with equality if and only if $\mathbf{U}$ is Gaussian.

2. (FII) For any two independent random vectors $\mathbf{U}, \mathbf{V}$ and any $0 \leq \lambda \leq 1$, we have

$$
\lambda^{2} \mathbf{J}(\mathbf{U})+(1-\lambda)^{2} \mathbf{J}(\mathbf{V})-\mathbf{J}(\mathbf{U}+\mathbf{V}) \succeq \mathbf{0} .
$$


We now give the proof of Lemma 2. Denote by $\boldsymbol{\rho}_{X+Z}(\cdot)$ and $\boldsymbol{\rho}_{X+\widetilde{Z}}(\cdot)$ the score functions for $\mathbf{X}+\mathbf{Z}$ and $\mathbf{X}+\widetilde{\mathbf{Z}}$, respectively and let

$$
\begin{aligned}
& \mathbf{N}:=\boldsymbol{\rho}_{X+Z}(\mathbf{X}+\mathbf{Z})+\left(\mathbf{K}_{X}+\mathbf{K}_{Z}\right)^{-1}(\mathbf{X}+\mathbf{Z}), \\
& \widetilde{\mathbf{N}}:=\boldsymbol{\rho}_{X+\widetilde{Z}}(\mathbf{X}+\widetilde{\mathbf{Z}})+\left(\mathbf{K}_{X}+\mathbf{K}_{\widetilde{Z}}\right)^{-1}(\mathbf{X}+\widetilde{\mathbf{Z}}) .
\end{aligned}
$$

For any matrix $\mathbf{B}$, we have

$$
\operatorname{Tr}\left(\mathbb{E}\left[\left(\mathbf{B N}-\widetilde{\mathbf{A}}^{1 / 2} \widetilde{\mathbf{N}}\right)\left(\mathbf{B N}-\widetilde{\mathbf{A}}^{1 / 2} \widetilde{\mathbf{N}}\right)^{t}\right]\right) \geq 0 .
$$

To expand the left-hand side of (103), we need to first calculate the correlations among random vectors $\boldsymbol{\rho}_{X+Z}(\mathbf{X}+\mathbf{Z}), \boldsymbol{\rho}_{X+\widetilde{Z}}(\mathbf{X}+\widetilde{\mathbf{Z}}), \mathbf{X}+\mathbf{Z}$ and $\mathbf{X}+\widetilde{\mathbf{Z}}$.

First, by the definition of Fisher information matrix and the Stein identity, we have

$$
\begin{aligned}
\mathbb{E}\left[\boldsymbol{\rho}_{X+Z}(\mathbf{X}+\mathbf{Z}) \boldsymbol{\rho}_{X+Z}^{t}(\mathbf{X}+\mathbf{Z})\right] & =\mathbf{J}(\mathbf{X}+\mathbf{Z}) \\
\mathbb{E}\left[\boldsymbol{\rho}_{X+Z}(\mathbf{X}+\mathbf{Z})(\mathbf{X}+\mathbf{Z})^{t}\right] & =-\mathbf{I} .
\end{aligned}
$$

Second, by the assumption $\mathbf{K}_{Z} \succeq \mathbf{K}_{\widetilde{Z}}$, we may write $\mathbf{Z}=\widetilde{\mathbf{Z}}+\widehat{\mathbf{Z}}$ where $\widehat{\mathbf{Z}}$ is a Gaussian vector with covariance matrix $\mathbf{K}_{\widehat{Z}}$ and independent of $\widetilde{\mathbf{Z}}$ and $\mathbf{X}$. By the convolution behavior of score function, we have

$$
\begin{aligned}
\mathbb{E}\left[\boldsymbol{\rho}_{X+Z}(\mathbf{X}+\mathbf{Z}) \boldsymbol{\rho}_{X+\widetilde{Z}}^{t}(\mathbf{X}+\widetilde{\mathbf{Z}})\right] \\
\quad=\mathbb{E}\left[\boldsymbol{\rho}_{X+\widetilde{Z}+\widehat{Z}}(\mathbf{X}+\widetilde{\mathbf{Z}}+\widehat{\mathbf{Z}}) \boldsymbol{\rho}_{X+\widetilde{Z}}^{t}(\mathbf{X}+\widetilde{\mathbf{Z}})\right] \\
\quad=\mathbb{E}\left[\boldsymbol{\rho}_{X+\widetilde{Z}+\widehat{Z}}(\mathbf{X}+\widetilde{\mathbf{Z}}+\widehat{\mathbf{Z}}) \mathbb{E}\left[\boldsymbol{\rho}_{X+\widetilde{Z}}^{t}(\mathbf{X}+\widetilde{\mathbf{Z}}) \mid \mathbf{X}+\widetilde{\mathbf{Z}}+\widehat{\mathbf{Z}}\right]\right] \\
\quad=\mathbb{E}\left[\boldsymbol{\rho}_{X+\widetilde{Z}+\widehat{Z}}(\mathbf{X}+\widetilde{\mathbf{Z}}+\widehat{\mathbf{Z}}) \boldsymbol{\rho}_{X+\widetilde{Z}+\widehat{Z}}^{t}(\mathbf{X}+\widetilde{\mathbf{Z}}+\widehat{\mathbf{Z}})\right] \\
\quad=\mathbf{J}(\mathbf{X}+\widetilde{\mathbf{Z}}+\widehat{\mathbf{Z}}) \\
\quad=\mathbf{J}(\mathbf{X}+\mathbf{Z}) .
\end{aligned}
$$

Third, we have

$$
\begin{aligned}
\mathbb{E}\left[\boldsymbol{\rho}_{X+\mathbf{Z}}(\mathbf{X}+\mathbf{Z})(\mathbf{X}+\widetilde{\mathbf{Z}})^{t}\right] & =\mathbb{E}\left[\boldsymbol{\rho}_{X+Z}(\mathbf{X}+\mathbf{Z})(\mathbf{X}+\mathbf{Z}-\widehat{\mathbf{Z}})^{t}\right] \\
& =-\mathbf{I}-\mathbb{E}\left[\boldsymbol{\rho}_{X+Z}(\mathbf{X}+\mathbf{Z}) \widehat{\mathbf{Z}}^{t}\right]
\end{aligned}
$$

where the last inequality follows from (105). Assume that $\mathbf{K}_{\widehat{Z}}$ is strictly positive definite. We have

$$
\widehat{\mathbf{z}}=-\mathbf{K}_{\widehat{Z}} \boldsymbol{\rho}_{\widehat{Z}}(\widehat{\mathbf{z}})
$$


because $\widehat{\mathbf{Z}}$ is Gaussian and, without loss of generality, can be assumed to have zero mean. It is then followed by

$$
\begin{aligned}
\mathbb{E}\left[\boldsymbol{\rho}_{X+\mathbf{Z}}(\mathbf{X}+\mathbf{Z}) \widehat{\mathbf{Z}}^{t}\right] & =-\mathbb{E}\left[\boldsymbol{\rho}_{X+Z}(\mathbf{X}+\mathbf{Z}) \boldsymbol{\rho}_{\widehat{Z}}^{t}(\widehat{\mathbf{Z}})\right] \mathbf{K}_{\widehat{\mathbf{Z}}} \\
& =-\mathbb{E}\left[\boldsymbol{\rho}_{X+\widetilde{Z}+\widehat{Z}}(\mathbf{X}+\widetilde{\mathbf{Z}}+\widehat{\mathbf{Z}}) \mathbb{E}\left[\boldsymbol{\rho}_{\widehat{Z}}^{t}(\widehat{\mathbf{Z}}) \mid \mathbf{X}+\widetilde{\mathbf{Z}}+\widehat{\mathbf{Z}}\right]\right] \mathbf{K}_{\widehat{\mathbf{Z}}} \\
& =-\mathbb{E}\left[\boldsymbol{\rho}_{X+\widetilde{Z}+\widehat{Z}}(\mathbf{X}+\widetilde{\mathbf{Z}}+\widehat{\mathbf{Z}}) \boldsymbol{\rho}_{X+\widetilde{Z}+\widehat{Z}}^{t}(\mathbf{X}+\widetilde{\mathbf{Z}}+\widehat{\mathbf{Z}})\right] \mathbf{K}_{\widehat{\mathbf{Z}}} \\
& =-\mathbf{J}(\mathbf{X}+\widetilde{\mathbf{Z}}+\widehat{\mathbf{Z}}) \mathbf{K}_{\widehat{\mathbf{Z}}} \\
& =-\mathbf{J}(\mathbf{X}+\mathbf{Z}) \mathbf{K}_{\widehat{\mathbf{Z}}}
\end{aligned}
$$

Note that even though in the above derivation we have assumed that $\mathbf{K}_{\widehat{\mathbf{Z}}}$ is strictly positive definite, we may verify (by using the definition of score function) that (118) holds for all positive semidefinite $\mathbf{K}_{\widehat{\mathbf{Z}}}$. Substituting (118) into (112), we obtain

$$
\mathbb{E}\left[\boldsymbol{\rho}_{X+\mathbf{Z}}(\mathbf{X}+\mathbf{Z})(\mathbf{X}+\widetilde{\mathbf{Z}})^{t}\right]=-\mathbf{I}+\mathbf{J}(\mathbf{X}+\mathbf{Z})\left(\mathbf{K}_{\mathbf{Z}}-\mathbf{K}_{\widetilde{\mathbf{Z}}}\right)
$$

Finally, we have

$$
\begin{aligned}
\mathbb{E}\left[\boldsymbol{\rho}_{X+\widetilde{Z}}(\mathbf{X}+\widetilde{\mathbf{Z}})(\mathbf{X}+\mathbf{Z})^{t}\right] & =\mathbb{E}\left[\boldsymbol{\rho}_{X+\widetilde{Z}}(\mathbf{X}+\widetilde{\mathbf{Z}})(\mathbf{X}+\widetilde{\mathbf{Z}}+\widehat{\mathbf{Z}})^{t}\right] \\
& =-\mathbf{I}+\mathbb{E}\left[\boldsymbol{\rho}_{X+\widetilde{Z}}(\mathbf{X}+\widetilde{\mathbf{Z}}) \widehat{\mathbf{Z}}^{t}\right] \\
& =-\mathbf{I}
\end{aligned}
$$

because $\widehat{\mathbf{Z}}$ is independent of both $\mathbf{X}$ and $\widetilde{\mathbf{Z}}$.

Substituting (104), (105), (110), (119) and (122) into the left-hand side of (103) and using the assumption $\mathbf{A}\left(\mathbf{K}_{X}+\mathbf{K}_{Z}\right)^{-1}=\widetilde{\mathbf{A}}\left(\mathbf{K}_{X}+\mathbf{K}_{\widetilde{Z}}\right)^{-1}$, we obtain

$$
\begin{aligned}
\operatorname{Tr} & \left(\mathbb{E}\left[\left(\mathbf{B N}-\widetilde{\mathbf{A}}^{1 / 2} \widetilde{\mathbf{N}}\right)\left(\mathbf{B N}-\widetilde{\mathbf{A}}^{1 / 2} \widetilde{\mathbf{N}}\right)^{t}\right]\right) \\
& =\operatorname{Tr}\left(\widetilde{\mathbf{A}}^{1 / 2} \mathbf{J}(\mathbf{X}+\widetilde{\mathbf{Z}})-\mathbf{A}^{1 / 2} \mathbf{J}(\mathbf{X}+\mathbf{Z})+\mathbf{f}(\mathbf{B})\left(\mathbf{J}(\mathbf{X}+\mathbf{Z})-\left(\mathbf{K}_{X}+\mathbf{K}_{Z}\right)^{-1}\right)\right)
\end{aligned}
$$

where

$$
\mathbf{f}(\mathbf{B}):=\mathbf{B}^{t} \mathbf{B}-\mathbf{B}^{t} \widetilde{\mathbf{A}}^{-1 / 2} \mathbf{A}-\mathbf{A} \widetilde{\mathbf{A}}^{-1 / 2} \mathbf{B}+\mathbf{A}
$$

is a matrix-valued quadratic function. We notice that $\mathbf{f}(\mathbf{B})=\mathbf{0}$ is equivalent to

$$
\left(\mathbf{B}-\widetilde{\mathbf{A}}^{-1 / 2} \mathbf{A}\right)^{t}\left(\mathbf{B}-\widetilde{\mathbf{A}}^{-1 / 2} \mathbf{A}\right)=\mathbf{A} \widetilde{\mathbf{A}}^{-1} \mathbf{A}-\mathbf{A} .
$$

By the assumption $\mathbf{A} \succeq \widetilde{\mathbf{A}}$, we have $\mathbf{A}^{-1} \preceq \widetilde{\mathbf{A}}^{-1}$ and hence

$$
\mathbf{A} \widetilde{\mathbf{A}}^{-1} \mathbf{A}-\mathbf{A}=\mathbf{A}\left(\widetilde{\mathbf{A}}^{-1}-\mathbf{A}^{-1}\right) \mathbf{A} \succeq \mathbf{0} .
$$

Therefore, the matrix quadratic equation $\mathbf{f}(\mathbf{B})=\mathbf{0}$ has at least two real solutions:

$$
\widetilde{\mathbf{A}}^{-1 / 2} \mathbf{A} \pm\left(\mathbf{A} \widetilde{\mathbf{A}}^{-1} \mathbf{A}-\mathbf{A}\right)^{1 / 2} .
$$


Picking $\mathbf{B}$ as one of them, we obtain from (123) the desired inequality (21).

The inequality (22) follows from (21) by letting

$$
\mathbf{A}=\mathbf{K}_{X}+\mathbf{K}_{Z}, \quad \widetilde{\mathbf{A}}=\mathbf{K}_{X}+\mathbf{K}_{\widetilde{Z}}
$$

This completes the proof of Lemma 2 .

\section{E Proof of Lemma 10}

Note that $\mathbf{Z}_{i}, i=1,2$, are Gaussian vectors and, without loss of generality, can be assumed to have zero mean. So we may write

$$
\mathbf{Z}_{i}=\sqrt{1-\lambda} \mathbf{Z}_{i, 1}+\sqrt{\lambda} \mathbf{Z}_{i, 2}
$$

where $\mathbf{Z}_{i, 1}$ and $\mathbf{Z}_{i, 2}$ are independent random vectors with the same distributions as that of $\mathbf{Z}_{i}$. We have

$$
\begin{aligned}
h\left(\mathbf{X}_{\lambda}+\mathbf{Z}_{i}\right) & =h\left(\sqrt{1-\lambda} \mathbf{X}_{0}+\sqrt{\lambda} \mathbf{X}_{G}^{*}+\mathbf{Z}_{i}\right) \\
& =h\left(\sqrt{1-\lambda}\left(\mathbf{X}_{0}+\mathbf{Z}_{i, 1}\right)+\sqrt{\lambda}\left(\mathbf{X}_{G}^{*}+\mathbf{Z}_{i, 2}\right)\right) \\
& =h\left(\mathbf{X}_{0}+\mathbf{Z}_{i, 1}+\sqrt{\frac{\lambda}{1-\lambda}}\left(\mathbf{X}_{G}^{*}+\mathbf{Z}_{i, 2}\right)\right)+\frac{n}{2} \log _{e}(1-\lambda) .
\end{aligned}
$$

Since both $\mathbf{X}_{G}^{*}$ and $\mathbf{Z}_{i, 2}$ are Gaussian, by the vector DeBruijn identity [10, Theorem 14], we have

$$
\begin{aligned}
\frac{d}{d \lambda} & h\left(\mathbf{X}_{\lambda}+\mathbf{Z}_{i}\right) \\
& =\frac{1}{2(1-\lambda)^{2}} \operatorname{Tr}\left(\left(\mathbf{K}_{X}^{*}+\mathbf{K}_{Z_{i}}\right) \mathbf{J}\left(\mathbf{X}_{0}+\mathbf{Z}_{i, 1}+\sqrt{\frac{\lambda}{1-\lambda}}\left(\mathbf{X}_{G}^{*}+\mathbf{Z}_{i, 2}\right)\right)\right)-\frac{n}{2(1-\lambda)} \\
& =\frac{1}{2(1-\lambda)} \operatorname{Tr}\left(\left(\mathbf{K}_{X}^{*}+\mathbf{K}_{Z_{i}}\right) \mathbf{J}\left(\sqrt{1-\lambda}\left(\mathbf{X}_{0}+\mathbf{Z}_{i, 1}\right)+\sqrt{\lambda}\left(\mathbf{X}_{G}^{*}+\mathbf{Z}_{i, 2}\right)\right)\right)-\frac{n}{2(1-\lambda)} \\
& =\frac{1}{2(1-\lambda)}\left(\operatorname{Tr}\left(\left(\mathbf{K}_{X}^{*}+\mathbf{K}_{Z_{i}}\right) \mathbf{J}\left(\mathbf{X}_{\lambda}+\mathbf{Z}_{i}\right)\right)-n\right) .
\end{aligned}
$$

It is then followed by

$$
\begin{aligned}
& \frac{d g(\lambda)}{d \lambda} \\
& \quad=\frac{d}{d \lambda}\left(h\left(\mathbf{X}_{\lambda}+\mathbf{Z}_{1}\right)-\mu h\left(\mathbf{X}_{\lambda}+\mathbf{Z}_{2}\right)\right) \\
& \quad=\frac{1}{2(1-\lambda)}\left(\operatorname{Tr}\left(\left(\mathbf{K}_{X}^{*}+\mathbf{K}_{Z_{1}}\right) \mathbf{J}\left(\mathbf{X}_{\lambda}+\mathbf{Z}_{1}\right)-\mu\left(\mathbf{K}_{X}^{*}+\mathbf{K}_{Z_{2}}\right) \mathbf{J}\left(\mathbf{X}_{\lambda}+\mathbf{Z}_{2}\right)\right)+n(\mu-1)\right) .(13
\end{aligned}
$$


The right-hand side of (137) can be evaluated as follows. First, by the assumption $\mathbf{M}_{1}=0, \mathbf{Z}_{1}$ and $\widetilde{\mathbf{Z}}_{1}$ have the same distribution. Therefore, we have

$$
\left(\mathbf{K}_{X}^{*}+\mathbf{K}_{Z_{1}}\right) \mathbf{J}\left(\mathbf{X}_{\lambda}+\mathbf{Z}_{1}\right)=\left(\mathbf{K}_{X}^{*}+\mathbf{K}_{\widetilde{Z}_{1}}\right) \mathbf{J}\left(\mathbf{X}_{\lambda}+\widetilde{\mathbf{Z}}_{1}\right)=(\mu-1)^{-1} \mathbf{K}_{\widetilde{Z}} \mathbf{J}\left(\mathbf{X}_{\lambda}+\widetilde{\mathbf{Z}}_{1}\right)
$$

where the last equality follows from (79) . Second, to apply Lemma 2, let us choose

$$
\mathbf{A}=\mathbf{K}_{X}^{*}+\mathbf{K}_{Z_{2}}, \quad \widetilde{\mathbf{A}}=\mathbf{K}_{X}^{*}+\mathbf{K}_{\widetilde{Z}_{2}} .
$$

Clearly, $\mathbf{A} \succeq \widetilde{\mathbf{A}}$ because $\mathbf{K}_{Z_{2}} \succeq \mathbf{K}_{\widetilde{Z}_{2}}$. Furthermore, denote the covariance matrix of $\mathbf{X}_{\lambda}$ by $\mathbf{K}_{X_{\lambda}}$ and we have

$$
\begin{aligned}
\widetilde{\mathbf{A}}^{-1}\left(\mathbf{K}_{X_{\lambda}}+\mathbf{K}_{\widetilde{Z}_{2}}\right) & =\left(\mathbf{K}_{X}^{*}+\mathbf{K}_{\widetilde{Z}_{2}}\right)^{-1}\left((1-\lambda) \mathbf{S}+\lambda \mathbf{K}_{X}^{*}+\mathbf{K}_{\widetilde{Z}_{2}}\right) \\
& =\left(\mathbf{K}_{X}^{*}+\mathbf{K}_{\widetilde{Z}_{2}}\right)^{-1}\left((1-\lambda)\left(\mathbf{S}+\mathbf{K}_{\widetilde{Z}_{2}}\right)+\lambda\left(\mathbf{K}_{X}^{*}+\mathbf{K}_{\widetilde{Z}_{2}}\right)\right) \\
& =(1-\lambda)\left(\mathbf{K}_{X}^{*}+\mathbf{K}_{\widetilde{Z}_{2}}\right)^{-1}\left(\mathbf{S}+\mathbf{K}_{\widetilde{Z}_{2}}\right)+\lambda \mathbf{I} \\
& =(1-\lambda)\left(\mathbf{K}_{X}^{*}+\mathbf{K}_{Z_{2}}\right)^{-1}\left(\mathbf{S}+\mathbf{K}_{Z_{2}}\right)+\lambda \mathbf{I} \\
& =\left(\mathbf{K}_{X}^{*}+\mathbf{K}_{Z_{2}}\right)^{-1}\left((1-\lambda)\left(\mathbf{S}+\mathbf{K}_{Z_{2}}\right)+\lambda\left(\mathbf{K}_{X}^{*}+\mathbf{K}_{Z_{2}}\right)\right) \\
& =\left(\mathbf{K}_{X}^{*}+\mathbf{K}_{\widetilde{Z}_{2}}\right)^{-1}\left((1-\lambda) \mathbf{S}+\lambda \mathbf{K}_{X}^{*}+\mathbf{K}_{\widetilde{Z}_{2}}\right) \\
& =\mathbf{A}^{-1}\left(\mathbf{K}_{X_{\lambda}}+\mathbf{K}_{Z_{2}}\right)
\end{aligned}
$$

where (143) follows from Lemma 9 . Applying Lemma 2, we obtain

$$
\begin{aligned}
\operatorname{Tr}\left(\left(\mathbf{K}_{X}^{*}+\mathbf{K}_{Z_{2}}\right) \mathbf{J}\left(\mathbf{X}_{\lambda}+\mathbf{Z}_{2}\right)\right) & \leq \operatorname{Tr}\left(\left(\mathbf{K}_{X}^{*}+\mathbf{K}_{\widetilde{Z}_{2}}\right) \mathbf{J}\left(\mathbf{X}_{\lambda}+\widetilde{\mathbf{Z}}_{2}\right)\right) \\
& =\mu(\mu-1)^{-1} \operatorname{Tr}\left(\mathbf{K}_{\widetilde{Z}} \mathbf{J}\left(\mathbf{X}_{\lambda}+\widetilde{\mathbf{Z}}_{1}+\widetilde{\mathbf{Z}}\right)\right)
\end{aligned}
$$

where the second equality follows from (79). Finally, we have

$$
n=\operatorname{Tr}(\mathbf{I})=\operatorname{Tr}\left(\mathbf{K}_{\widetilde{\mathbf{Z}}} \mathbf{J}(\widetilde{\mathbf{Z}})\right) .
$$

because $\widetilde{Z}$ is Gaussian. Substituting (138), (148) and (149) into (137), we obtain

$$
\frac{d g(\lambda)}{d \lambda} \geq \frac{\mu^{2}}{2(1-\lambda)(\mu-1)} \operatorname{Tr}\left(\mathbf{K}_{\widetilde{Z}}\left(\mu^{-2} \mathbf{J}\left(\mathbf{X}_{\lambda}+\widetilde{\mathbf{Z}}_{1}\right)+\left(1-\mu^{-1}\right)^{2} \mathbf{J}(\widetilde{\mathbf{Z}})-\mathbf{J}\left(\mathbf{X}_{\lambda}+\widetilde{\mathbf{Z}}_{1}+\widetilde{\mathbf{Z}}\right)\right)\right.
$$

Now by the classical FII, for any $\mu \geq 1$, we have

$$
\left.\mu^{-2} \mathbf{J}\left(\mathbf{X}_{\lambda}+\widetilde{\mathbf{Z}}_{1}\right)+\left(1-\mu^{-1}\right)^{2} \mathbf{J}(\widetilde{\mathbf{Z}})-\mathbf{J}\left(\mathbf{X}_{\lambda}+\widetilde{\mathbf{Z}}_{1}+\widetilde{\mathbf{Z}}\right)\right) \succeq \mathbf{0}
$$

It is followed by

$$
\begin{aligned}
\operatorname{Tr} & \left(\mathbf{K}_{\widetilde{Z}}\left(\mu^{-2} \mathbf{J}\left(\mathbf{X}_{\lambda}+\widetilde{\mathbf{Z}}_{1}\right)+\left(1-\mu^{-1}\right)^{2} \mathbf{J}(\widetilde{\mathbf{Z}})-\mathbf{J}\left(\mathbf{X}_{\lambda}+\widetilde{\mathbf{Z}}_{1}+\widetilde{\mathbf{Z}}\right)\right)\right) \\
& =\operatorname{Tr}\left(\mathbf{K}_{\widetilde{Z}}^{1 / 2}\left(\mu^{-2} \mathbf{J}\left(\mathbf{X}_{\lambda}+\widetilde{\mathbf{Z}}_{1}\right)+\left(1-\mu^{-1}\right)^{2} \mathbf{J}(\widetilde{\mathbf{Z}})-\mathbf{J}\left(\mathbf{X}_{\lambda}+\widetilde{\mathbf{Z}}_{1}+\widetilde{\mathbf{Z}}\right)\right) \mathbf{K}_{\widetilde{Z}}^{1 / 2}\right) \\
& \geq 0 .
\end{aligned}
$$

Substituting (153) into (150), we conclude that that $d g(\lambda) / d \lambda \geq 0$ for all $0 \leq \lambda \leq 1$ and all $\mu \geq 1$. This completes the proof of Lemma 10. 


\section{F Proof of Corollary [5]}

By Theorem 1, for any random vector $\mathbf{X}$ in $\mathcal{R}^{2}$ satisfying $\operatorname{Cov}(\mathbf{X}) \leq \mathbf{S}$ and any $\mu \geq 1$, we have

$h\left(\mathbf{X}+\mathbf{Z}_{1}\right)-\mu h\left(\mathbf{X}+\mathbf{Z}_{2}\right) \leq \max _{\mathbf{0} \preceq \mathbf{K}_{X} \preceq \mathbf{S}}\left(\frac{1}{2} \log _{e}(2 \pi e)^{2}\left|\mathbf{K}_{X}+\mathbf{K}_{Z_{1}}\right|-\frac{\mu}{2} \log _{e}(2 \pi e)^{2}\left|\mathbf{K}_{X}+\mathbf{K}_{Z_{2}}\right|\right)$.

Appending $\mu h\left(\mathbf{Z}_{2}\right)-h\left(\mathbf{Z}_{1}\right)$ to both sides of (154), we obtain

$$
I\left(\mathbf{X} ; \mathbf{X}+\mathbf{Z}_{1}\right)-\mu I\left(\mathbf{X} ; \mathbf{X}+\mathbf{Z}_{2}\right) \leq \max _{\mathbf{0} \preceq \mathbf{K}_{X} \preceq \mathbf{S}}\left(\frac{1}{2} \log _{e}\left|\mathbf{I}+\mathbf{K}_{Z_{1}}^{-1} \mathbf{K}_{X}\right|-\frac{\mu}{2} \log _{e}\left|\mathbf{I}+\mathbf{K}_{Z_{2}}^{-1} \mathbf{K}_{X}\right|\right) .
$$

For $i=1,2$, the eigenvalue decomposition of $\mathbf{K}_{Z_{i}}$ can be written as $\mathbf{K}_{Z_{i}}=\mathbf{V}_{i} \boldsymbol{\Sigma}_{i} \mathbf{V}_{i}^{t}$ where $\mathbf{V}_{i}=\left(\mathbf{v}_{i 1}, \mathbf{v}_{i 2}\right)$ is a $2 \times 2$ orthonormal matrix and $\boldsymbol{\Sigma}_{i}=\operatorname{Diag}\left(\lambda_{i 1}, \lambda_{i 2}\right)$ is a $2 \times 2$ diagonal matrix. Next we consider taking limits on both sides of (155) as $\lambda_{12}, \lambda_{21} \rightarrow \infty$.

First consider the left-hand side of (155). Let $\overline{\mathbf{Z}}_{i}=\left(\bar{Z}_{i 1}, \bar{Z}_{i 2}\right)^{t}:=\mathbf{V}_{i}^{t} \mathbf{Z}_{i}$. We have $\operatorname{Cov}\left(\overline{\mathbf{Z}}_{i}\right)=\boldsymbol{\Sigma}_{i}$, i.e., $\overline{\mathbf{Z}}_{i}$ is a white Gaussian vector. Since $\mathbf{V}_{i}$ is invertible, we have

$$
I\left(\mathbf{X} ; \mathbf{X}+\mathbf{Z}_{i}\right)=I\left(\mathbf{V}_{i}^{t} \mathbf{X} ; \mathbf{V}_{i}^{t} \mathbf{X}+\overline{\mathbf{Z}}_{i}\right) .
$$

We have the following simple lemma.

Lemma 14 Let $\mathbf{Z}=\left(Z_{1}, Z_{2}\right)^{t}$ where $Z_{1}$ and $Z_{2}$ are two independent Gaussian variables with variance $\sigma_{1}^{2}$ and $\sigma_{2}^{2}$, respectively. For any random vector $\mathbf{X}=\left(X_{1}, X_{2}\right)^{t}$ independent of $\mathbf{Z}$, we have

$$
\lim _{\sigma_{2}^{2} \rightarrow \infty} I(\mathbf{X} ; \mathbf{X}+\mathbf{Z})=I\left(X_{1} ; X_{1}+Z_{1}\right)
$$

Proof: By the chain rule of mutual information, we have

$$
\begin{aligned}
I(\mathbf{X} ; \mathbf{X}+\mathbf{Z}) & =I\left(X_{1} ; \mathbf{X}+\mathbf{Z}\right)+I\left(X_{2} ; \mathbf{X}+\mathbf{Z} \mid X_{1}\right) \\
& =I\left(X_{1} ; X_{1}+Z_{1}\right)+I\left(X_{1} ; X_{2}+Z_{2} \mid X_{1}+Z_{1}\right)+I\left(X_{2} ; \mathbf{X}+\mathbf{Z} \mid X_{1}\right) .
\end{aligned}
$$

Next we show that both $I\left(X_{1} ; X_{2}+Z_{2} \mid X_{1}+Z_{1}\right)$ and $I\left(X_{2} ; \mathbf{X}+\mathbf{Z} \mid X_{1}\right)$ tend to zero in the limit as $\sigma_{2}^{2} \rightarrow \infty$, and thus the desired result (157) will follow.

First,

$$
I\left(X_{1} ; X_{2}+Z_{2} \mid X_{1}+Z_{1}\right) \leq I\left(X_{1} ; X_{2}+Z_{2}\right) \leq I\left(X_{2} ; X_{2}+Z_{2}\right)
$$


where the inequalities are due to the Markov chain $X_{1}+Z_{1} \leftrightarrow X_{1} \leftrightarrow X_{2}+Z_{2}$ and $X_{1} \leftrightarrow$ $X_{2} \leftrightarrow X_{2}+Z_{2}$, respectively. Second,

$$
\begin{aligned}
I\left(X_{2} ; \mathbf{X}+\mathbf{Z} \mid X_{1}\right) & =I\left(X_{2} ; X_{2}+Z_{2} \mid X_{1}\right)+I\left(X_{2} ; X_{1}+Z_{1} \mid X_{1}, X_{2}+Z_{2}\right) \\
& =I\left(X_{2} ; X_{2}+Z_{2} \mid X_{1}\right)+I\left(X_{2} ; Z_{1} \mid X_{1}, X_{2}+Z_{2}\right) \\
& =I\left(X_{2} ; X_{2}+Z_{2} \mid X_{1}\right) \\
& \leq I\left(X_{2} ; X_{2}+Z_{2}\right)
\end{aligned}
$$

where (163) follows from the fact that $Z_{1}$ is independent of $Z_{2}$ and $\mathbf{X}$ so $I\left(X_{2} ; Z_{1} \mid X_{1}, X_{2}+\right.$ $\left.Z_{2}\right)=0$, and (164) is due to the Markov chain $X_{1} \rightarrow X_{2} \rightarrow X_{2}+Z_{2}$. By the fact

$$
\lim _{\sigma_{2}^{2} \rightarrow \infty} I\left(X_{2} ; X_{2}+Z_{2}\right)=0
$$

we have from (160) and (164) that both $I\left(X_{1} ; X_{2}+Z_{2} \mid X_{1}+Z_{1}\right)$ and $I\left(X_{2} ; \mathbf{X}+\mathbf{Z} \mid X_{1}\right)$ tend to zero in the limit as $\sigma_{2}^{2} \rightarrow \infty$. This completes the proof of Lemma 14.

Applying Lemma 14 to (156), we obtain

$$
\begin{aligned}
\lim _{\lambda_{12} \rightarrow \infty} I\left(\mathbf{X} ; \mathbf{X}+\mathbf{Z}_{1}\right) & =I\left(\mathbf{v}_{11}^{t} \mathbf{X} ; \mathbf{v}_{11}^{t} \mathbf{X}+\bar{Z}_{11}\right) \\
\lim _{\lambda_{21} \rightarrow \infty} I\left(\mathbf{X} ; \mathbf{X}+\mathbf{Z}_{2}\right) & =I\left(\mathbf{v}_{22}^{t} \mathbf{X} ; \mathbf{v}_{22}^{t} \mathbf{X}+\bar{Z}_{22}\right)
\end{aligned}
$$

which gives

$$
\lim _{\lambda_{12}, \lambda_{21} \rightarrow \infty} I\left(\mathbf{X} ; \mathbf{X}+\mathbf{Z}_{1}\right)-\mu I\left(\mathbf{X}+\mathbf{Z}_{2}\right)=I\left(\mathbf{v}_{11}^{t} \mathbf{X} ; \mathbf{v}_{11}^{t} \mathbf{X}+\bar{Z}_{11}\right)-\mu I\left(\mathbf{v}_{22}^{t} \mathbf{X} ; \mathbf{v}_{22}^{t} \mathbf{X}+\bar{Z}_{22}\right) .
$$

Next, let us consider the right-hand side of (155). For any semidefinite $\mathbf{K}_{X}$, we have

$$
\begin{aligned}
\lim _{\lambda_{12}, \lambda_{21} \rightarrow \infty} & \frac{1}{2} \log _{e}\left|\mathbf{I}+\mathbf{K}_{Z_{1}}^{-1} \mathbf{K}_{X}\right|-\frac{\mu}{2} \log _{e}\left|\mathbf{I}+\mathbf{K}_{Z_{2}}^{-1} \mathbf{K}_{X}\right| \\
= & \lim _{\lambda_{12}, \lambda_{21} \rightarrow \infty} \frac{1}{2} \log _{e}\left|\mathbf{I}+\boldsymbol{\Sigma}_{1}^{-1} \mathbf{V}_{1}^{t} \mathbf{K}_{X} \mathbf{V}_{1}\right|-\frac{\mu}{2} \log _{e}\left|\mathbf{I}+\boldsymbol{\Sigma}_{2}^{-1} \mathbf{V}_{2}^{t} \mathbf{K}_{X} \mathbf{V}_{2}\right| \\
& =\frac{1}{2} \log _{e}\left(1+\lambda_{11}^{-1} \mathbf{v}_{11}^{t} \mathbf{K}_{X} \mathbf{v}_{11}\right)-\frac{\mu}{2} \log _{e}\left(1+\lambda_{22}^{-1} \mathbf{v}_{22}^{t} \mathbf{K}_{X} \mathbf{V}_{22}\right)
\end{aligned}
$$

due to the continuity of the function $f(\mathbf{A})=\log _{e}|\mathbf{I}+\mathbf{A}|$ over the semidefinite matrix $\mathbf{A}$. Furthermore, the continuity is uniform over semidefinite matrices which are bounded from above by some strictly positive semidefinite matrix. Therefore, we have

$$
\begin{aligned}
& \lim _{\lambda_{12}, \lambda_{21} \rightarrow \infty} \max _{\mathbf{0} \preceq \mathbf{K}_{X} \preceq \mathbf{S}}\left(\frac{1}{2} \log _{e}\left|\mathbf{I}+\mathbf{K}_{Z_{1}}^{-1} \mathbf{K}_{X}\right|-\frac{\mu}{2} \log _{e}\left|\mathbf{I}+\mathbf{K}_{Z_{2}}^{-1} \mathbf{K}_{X}\right|\right) \\
& \quad=\max _{\mathbf{0} \preceq \mathbf{K}_{X} \preceq \mathbf{S}}\left(\frac{1}{2} \log _{e}\left(1+\lambda_{11}^{-1} \mathbf{v}_{11}^{t} \mathbf{K}_{X} \mathbf{v}_{11}\right)-\frac{\mu}{2} \log _{e}\left(1+\lambda_{22}^{-1} \mathbf{v}_{22}^{t} \mathbf{K}_{X} \mathbf{v}_{22}\right)\right) .
\end{aligned}
$$


Substituting (168) and (171) into (154), we obtain

$$
\begin{aligned}
& I\left(\mathbf{v}_{11}^{t} \mathbf{X} ; \mathbf{v}_{11}^{t} \mathbf{X}+\bar{Z}_{11}\right)-\mu I\left(\mathbf{v}_{22}^{t} \mathbf{X} ; \mathbf{v}_{22}^{t} \mathbf{X}+\bar{Z}_{22}\right) \\
& \quad \leq \max _{\mathbf{0} \preceq \mathbf{K}_{X} \preceq \mathbf{S}}\left(\frac{1}{2} \log _{e}\left(1+\lambda_{11}^{-1} \mathbf{v}_{11}^{t} \mathbf{K}_{X} \mathbf{v}_{11}\right)-\frac{\mu}{2} \log _{e}\left(1+\lambda_{22}^{-1} \mathbf{v}_{22}^{t} \mathbf{K}_{X} \mathbf{v}_{22}\right)\right)
\end{aligned}
$$

and hence

$$
\begin{aligned}
& h\left(\mathbf{v}_{11}^{t} \mathbf{X}+\bar{Z}_{11}\right)-\mu h\left(\mathbf{v}_{22}^{t} \mathbf{X}+\bar{Z}_{22}\right) \\
& \quad \leq \max _{\mathbf{0} \preceq \mathbf{K}_{X} \preceq \mathbf{S}}\left(\frac{1}{2} \log _{e}\left(2 \pi e\left(\mathbf{v}_{11}^{t} \mathbf{K}_{X} \mathbf{v}_{11}+\lambda_{11}\right)\right)-\frac{\mu}{2} \log _{e}\left(2 \pi e\left(\mathbf{v}_{22}^{t} \mathbf{K}_{X} \mathbf{v}_{22}+\lambda_{22}\right)\right)\right)
\end{aligned}
$$

for any random vector $\mathbf{X}$ in $\mathcal{R}^{2}$ satisfying $\operatorname{Cov}(\mathbf{X}) \preceq \mathbf{S}$ and any $\mu \geq 1$. Corollary 5 thus follows from (173) and the fact that only the marginal distributions of $Z_{11}$ and $Z_{22}$ matter in the problem.

\section{G Proof of Corollary [ 6}

Let $\mathbf{v}_{1}=(1,1)^{t}$ and $\mathbf{v}_{2}=(0,1)^{t}$, and take into account

$$
\left\{\mathbf{X}: \operatorname{Var}(\mathbf{X}) \leq a_{1}\right\}=\bigcup_{\mathbf{S}:(\mathbf{S})_{11}=a_{1}}\{\mathbf{X}: \operatorname{Cov}(\mathbf{X}) \preceq \mathbf{S}\}
$$

By Corollary [5, the optimization problem

$$
\begin{array}{ll}
\max _{X_{1}, X_{2}} & h\left(X_{1}+X_{2}+Z\right)-\mu h\left(X_{2}+Z\right) \\
\text { subject to } & \operatorname{Var}\left(X_{1}\right) \leq a_{1}
\end{array}
$$

where the maximization is over all jointly distributed random variables independent of $Z$ has a Gaussian optimal solution for all $\mu \geq 1$. Let $\left(X_{1 G}^{*}, X_{2 G}^{*}\right)$ be the optimal Gaussian solution of the optimization problem (175). We have

$$
h\left(X_{1}+X_{2}+Z\right)-\mu h\left(X_{2}+Z\right) \leq h\left(X_{1 G}^{*}+X_{2 G}^{*}+Z\right)-\mu h\left(X_{2 G}^{*}+Z\right)
$$

for any jointly distributed random variables $\left(X_{1}, X_{2}\right)$ such that $\operatorname{Var}\left(X_{1}\right) \leq a_{1}$.

For $\mu=\infty$, we have $h\left(X_{2 G}^{*}+Z\right)=h(Z)$; for $\mu=1$, we have $h\left(X_{2 G}^{*}+Z\right)=a_{2}^{*}$ where $a_{2}^{*}$ is defined in (35). By the intermediate value theorem, for any $h(Z) \leq a_{2} \leq a_{2}^{*}$, there exists a $\mu$ such that $h\left(X_{2 G}^{*}+Z\right)=a_{2}$. Thus, for any jointly distributed random variables $\left(X_{1}, X_{2}\right)$ such that $\operatorname{Var}\left(X_{1}\right) \leq a_{1}$ and $h\left(X_{2}+Z\right) \leq a_{2}$, we have by (176)

$$
\begin{aligned}
h\left(X_{1}+X_{2}+Z\right) & \leq h\left(X_{1 G}^{*}+X_{2 G}^{*}+Z\right)+\mu\left(h\left(X_{2}+Z\right)-h\left(X_{2 G}^{*}+Z\right)\right) \\
& \leq h\left(X_{1 G}^{*}+X_{2 G}^{*}+Z\right) .
\end{aligned}
$$

We conclude that the optimization problem (34) has a Gaussian optimal solution for all $a_{1} \geq 0$ and all $h(Z) \leq a_{2} \leq a_{2}^{*}$. This completes the proof of Corollary 6 . 


\section{References}

[1] C. E. Shannon, "A mathematical theory of communication," Bell Syst. Tech. J., vol. 27, pp. 623-656, Oct. 1948.

[2] A. J. Stam, "Some inequalities satisfied by the quantities of information of Fisher and Shannon," Info. Ctrl., vol. 2, no. 2, pp. 101-112, Jun. 1959.

[3] N. M. Blachman, "The convolution inequality for entropy powers", IEEE Trans. Info. Theory, vol. IT-11, no, 2, pp. 267-271, Apr. 1965.

[4] P. P. Bergman, "A simple converse for broadcast channels with additive white Gaussian noise," IEEE Trans. Info. Theory, vol. IT-20, no. 2, pp. 279-280, Mar. 1974.

[5] Y. Oohama, "The rate-distortion function for the quadratic Gaussian CEO problem," IEEE Trans. Info. Theory, vol. 44, vol. 3, pp. 1057-1070, May 1998.

[6] L. Ozarow, "On a source coding problem with two channels and three receivers," Bell Syst. Tech. J., vol. 59, no. 10, pp. 1909-1921, Dec. 1980.

[7] K. Marton, "A coding theorem for the discrete memoryless broadcast channel," IEEE Trans. Info. Theory, vol. 44, vol. IT-25, pp. 306-311, May 1979.

[8] D. Slepian and J. K. Wolf, "Noiseless coding of correlated information sources," IEEE Trans. Info. Theory, vol. IT-19, no. 4, pp. 471-480, Jul. 1973.

[9] H. Weingarten, Y. Steinberg, and S. Shamai (Shitz), "The capacity region of the Gaussian MIMO broadcast channel," IEEE Trans. Info. Theory, submitted for publication.

[10] A. Dembo, T. M. Cover, and J. A. Thomas, "Information theoretic inequalities," IEEE Trans. Info. Theory, vol. 37, no. 6, pp. 1501-1518, Nov. 1991. D.

[11] T. M. Cover and Z. Zhang, "On the maximum entropy of the sum of two dependent random variables," IEEE Trans. Info. Theory, vol. 40, no. 4, pp. 1244-1246, Jul. 1994.

[12] S. Ihara, "On the capacity of channels with additive non-Gaussian noise," Info. Ctrl., vol. 37, no. 1, pp. 34-39, Apr. 1978.

[13] S. N. Diggavi and T. M. Cover, "The worst additive noise under a covariance constraint," IEEE Trans. Info. Theory, vol. 47, no. 7, pp. 3072-3081, Nov. 2001.

[14] R. Zamir, "A proof of the Fisher information inequality via a data processing argument," IEEE Trans. Info. Theory, vol. 44, no. 3, pp. 1246-1250, May 1998.

[15] D. Guo, S. Shamai (Shitz), and S. Verdú, "Additive non-gaussian noise channels: Mutual information and conditional mean estimation," in Proc. IEEE Int. Symp. Info. Theory, Adelaide, Australia, Sept. 2005. 
[16] H. Wang and P. Viswanath, "Vector Gaussian multiple description with individual and central receivers," IEEE Trans. Info. Theory, submitted for publication.

[17] R. Gallager, "Capacity and coding for degraded broadcast channels," Prob. Pered. Info., vol. 10, no. 3, pp. 3-14, Jul.-Sept. 1974.

[18] O. Johnson, Information Theory and The Central Limit Theorem, London: Imperial College Press, 2004. 